y

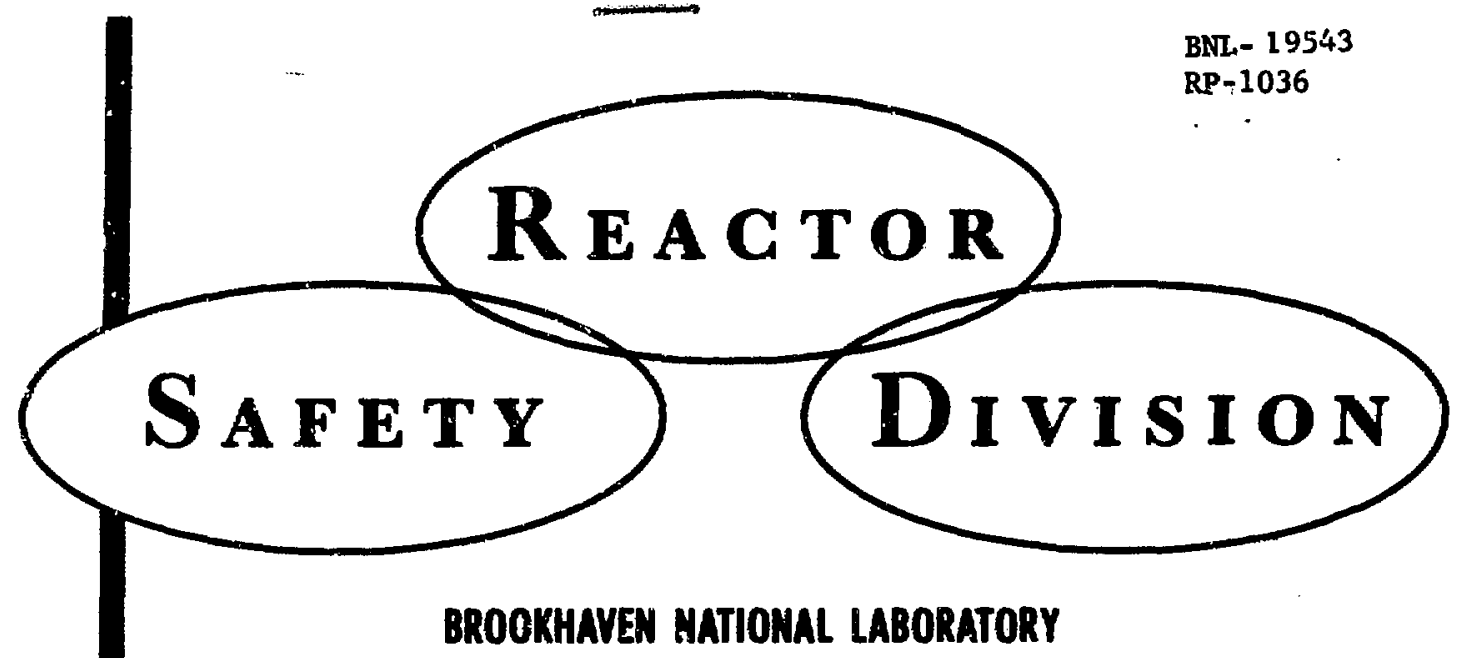

INCORPORATION OF EPITHERMAL PROTON

CHEMICAL BINDTNG EFFECTS IN HAMMER

Arthur Buslik and John Herczeg

November 1974

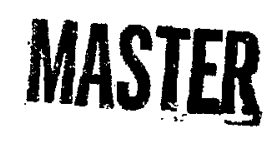

MASTER 


\section{INCORPORATION OF EPITHERMAL PROTON CHEMICAL BINDING EFFECTS IN HAMYER*}

- NOTICE

This report was prepared as an acabun of Hurk sponsared by the United States Government. Nitilher bie United States nor the United States Erierty Hesearch and Developmen ${ }^{*}$ Administration, nor any of their employees, nor any of theis contractors, subcontractors, or their employees, makes any wamanty, express or implied, or assumes any legal liability or responsibility for the accuracy, completencss or usefligess of any infurmation. apparatus, product ur process disclosed, or represents that its use would nut infringe privatcly owned rights.

Axthur Buslik and John Herczeg

\section{Introduction}

This reports those chang=s to the hydrogen eptthermal library In HAMER, and those changes to the HAMER program $(1,2)$ itself, which are required to incorporate the effects of the chemical binding and thermal motion of the protons in water on the epithermal neutron flux and reaction rates. The changes to the hydrogen epithermal 11 brary required are changes to the slowing-down power $\xi_{s}$, the Greuling-Goertzel parameter $Y$, and the P-1 component of the scattering cross-section ${ }_{-1}$. These changes are based on the kernel developed by Cady, Kirouac and McInerney.(3)

The changes to the HAMAER program which are required consist of changes to the program to ensure that the slowing-down power $\xi_{8}$ and the GreulingGoertzel parameter $Y$ for hydrogen are used in all of the subroutines (HAMER at present assumes in certain of its subroutines that the hydrogen slowing-down power $\xi^{\prime} s_{s}$ is equal to the $P-0$ component of the scattering

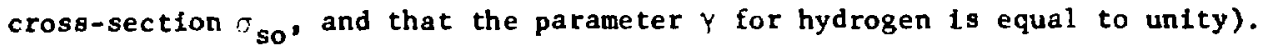
In addition, changes are required to modify the Dancoff factor uged in the Pu-240 l eV resonance treatment and the cosine current calculation of the lattice escape probabilities used on the resonance absorption calculation. These changes are along the lines discussed in references 7 and 8 .

Another approach to obtaining cross-sections for hydrogen which account for epithermal chemical binding effects is to obtain values of $5_{8}$ by multigroup so as to reproduce the Corngold asymptotic expansion fluxes exactly for plain water, and to leave the value of the Greuling-Goertzel parameter $Y$ equal to unity. This is the approach used by $J$. Hardy, Jr. (6) as suggested by E. M. C lbard. However, by also varying $Y$ (as is done in the method presented here), one would hope to get better accuracy in cases of heavy absorption. Moreover, this is more consistent with the changes made in the resonance absorption treatment for the Pu-240 $1 \mathrm{eV}$ resonance.

*Research carried out at Brookhaven National Laboratory under contract with the U. S. Atomic Energy Commission. 
The changes to the HAMER hyarogen epithermal library will firgt be presented, and a comparison of fluxes as calculated with these hydrogen cross-sections and parameters to fluxes as calculated by the Corngold asymptotic expansion $(4,5)$ will be presented. This comparison acts as a check on the correctuess of the hydrozen cross-sections and parameters obtained. (The comparisons do not check $\tau_{91}$. )

\section{The Changes to the HAMAER Epithermal Library}

The formulae used for the values of $\tau_{s}$ and $\psi$ for hydrogen are derived in reference 8 . These formulae are:

$$
\xi_{s}(E)=\left(1-\frac{1}{2} \frac{k T^{2}}{E}\right)^{2}
$$

and

$$
Y(E)=1-\frac{1}{2} \frac{k T^{*}}{E}
$$

Here $g$ is the asymptotic value of the hydrogen scattering cross-section, and was taken as 20.4 barns in the computations; $E$ is the neutron energy and $T *$ is the effective temperature associaced with the short collision time approximation ${ }^{(9)}$. The value of $T *$ used in the computations corresponds to a moderator temperacure of $500^{\circ} \mathrm{F}\left(=533^{\circ} \mathrm{K}\right)$ in a Nelkin kernel model. (10) The value of $T^{*}$ obtained is $1453^{\circ} \mathrm{K}$. One should note that the values of the hydrogen eptthermal library parameters are therefore dependent on the water temperature, and that the calculated parameters correspond to a water temperature of $500^{\circ} \mathrm{F}$. The temperature dependence of the parameters is rather weak, however, since $T *$ depends rather weakly on $T$. (At room temperature $\mathrm{T}^{*}$ is $1360^{\circ} \mathrm{K}$, while at $533^{\circ} \mathrm{K}, \mathrm{T}^{*}$ is $1453^{\circ} \mathrm{K}$. )

The value of $s$ (E) is the same as in an effective temperature kernel mocisl, and is given by

$$
\sigma_{5}(E)=\frac{2}{3} \sigma\left[1-\frac{3}{2} \frac{k T}{E}+\frac{5}{\sqrt{\pi}}\left(\frac{k T}{E}\right)^{\frac{3}{2}}-\frac{9}{4}\left(\frac{k T}{E}\right)^{x}\right]
$$

This formula was taken from the Cady, Kirouac and McInerney article. (3) 
The value of $\sigma_{80}(E) 18$ also the same as that for an effective temperature kernel and the formula for ${ }_{\text {so }}(\mathrm{E})$,

$$
r_{\text {so }}(E)=J\left(1+\frac{1}{2} \frac{k T^{\star}}{E}\right) \text {, }
$$

is also taken from reterence 3 .

Group averages of the various parameters were obtalned by taking the arithmetic average of the quantity at the upper and lower energies bounding the multigroup. For example,

$$
\bar{\sigma}_{s, j}=\frac{1}{2}\left[\sigma_{s 0}\left(E_{j+1}\right)+\sigma_{s 0}\left(E_{j}\right)\right]
$$

where $E_{j}$ and $E_{j+1}$ are the energles bounding the $j$ th multigroup.

The chemical binding effects were included for energies up to and including group 36; for energles greater than the upper cutpoint of this group, chemical binding effects were neglected and the values of the parameters used in the previous library for hydrogen were used.

The hydrogen cross-sections obtained are given in Table $I$. The 1sotope identity number is 2001001 , and the cross-sections are on tape N35838. Note ti:at the cross-sections in Table I are not in the same format as a printout of Lise HAMMER ept the rmal Iibrary -- some columns have been omicted and others have been rearranged. The column labelled "age number" 18 what we have denoted by $Y$. The omitted parameters (inelastic scatiering, fission crosssection, neutrons per fission) are all zero for hydrogen.

\section{Vertfication of the Hydrogen Cross-Section Changes}

In order to verify the cross-section changes made, the neutron fluxes in a medium composed of plain water (wth oxygen scattering effects neglected) were computed by means of the Corngold asymptotic expansion and compared to the flixes obtalned by running a HAMER problem using the new hydrogen 11brary. In addition, water poisoned with a $\frac{1}{v}$ absorber was considered. Five terms in the corngold asymptotic expansion were used. It turns out that to this order the expansion is Identical to an expansion derived in a much simpler fashion by Parks (11) (see also pages $103 \mathrm{ff}$ of reference 5). The 
TABLE I. Hydrogen cross-sections including binding effects.

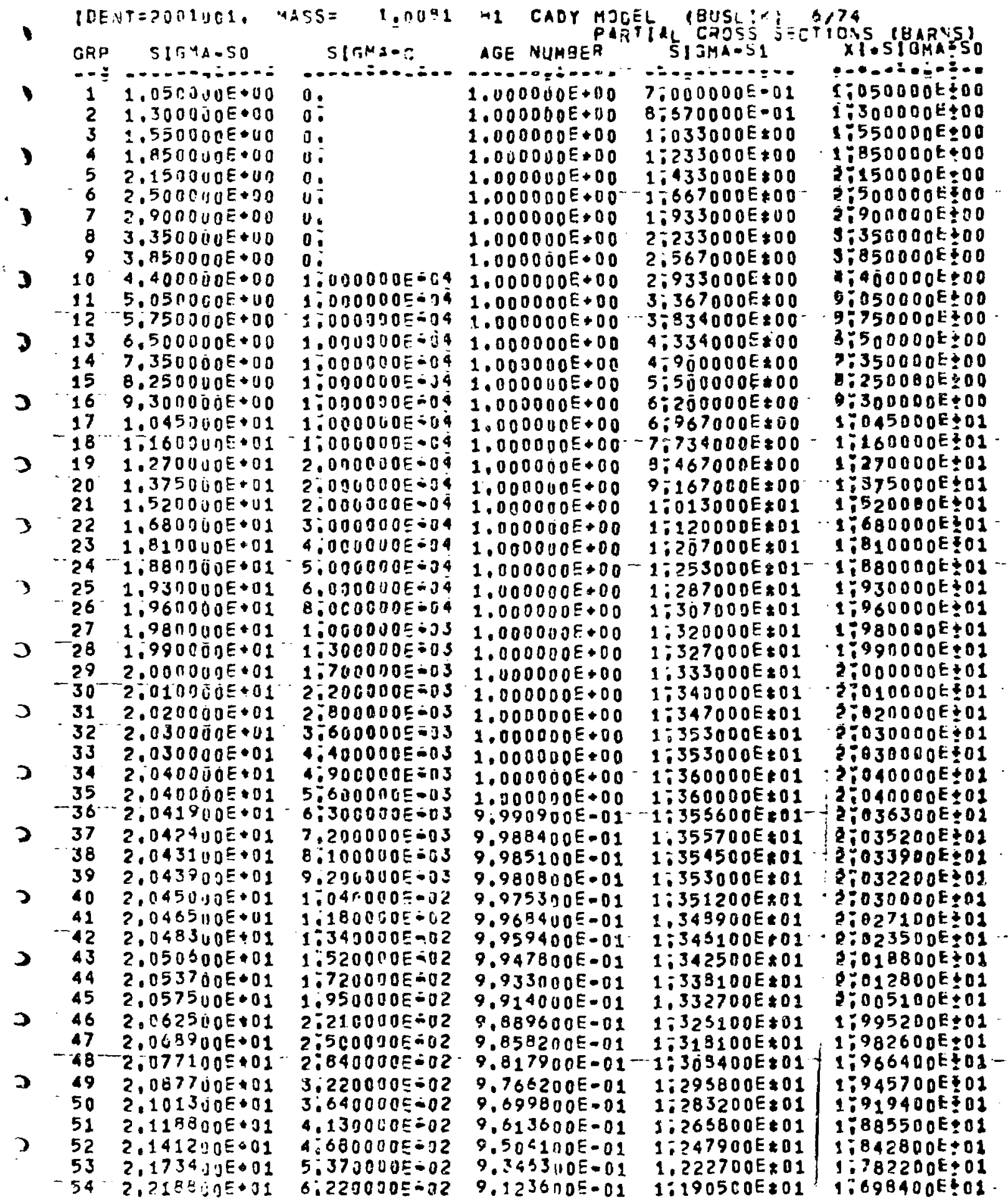

CAPTURE IVPESRAL $=1.334$ IJ $3 \div 31$

FISSIOV INTEGRAL $=0$. 
$-4-$

expansion, to the order given, way be written in the form

$$
\begin{aligned}
& E P(E)=1-\frac{3 \mu}{p}+\frac{6 \mu^{2}+1}{p^{2}}-\frac{10 \mu^{3}+4 R}{p^{3}} \\
& +\left[\frac{15}{4}+\frac{43}{4} \mu^{2}+15 \mu^{4}-\frac{1}{2} \frac{B r}{T^{2}}\right] \frac{1}{p^{4}}
\end{aligned}
$$

here

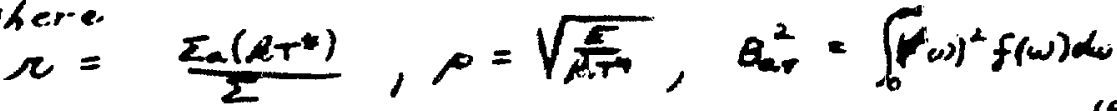

and the effective temperature is given by

$$
A r^{*}=\frac{1}{2} \int_{0}^{\infty} f(\omega) d \omega \cos \frac{\lambda \omega}{2 \pi} d \omega
$$

Here $f(u)$ Ls the phonon frequency spectrum; for the Welkin model $1 \mathrm{t}$ is given by

$$
f(\omega)=\sum_{i=1}^{4} \frac{1}{H_{i}} \delta\left(\omega-\omega_{i}\right)
$$

where

$$
\begin{aligned}
& A_{1}=18 \\
& A_{0}=2.32 \\
& A_{0}=5.84 \\
& A_{1}=2.92
\end{aligned}
$$

$$
\begin{aligned}
& t_{L_{2}}=0 \\
& H_{L_{1}}=.06 \mathrm{eV} \\
& t_{L_{3}}=.205 \mathrm{eV} \\
& t_{L_{4}}=.481 \mathrm{eV}
\end{aligned}
$$

It is assumed that the absorption cross-section has a $\frac{1}{v}$ energy dependpence; the quantity $\sum$ is the asymptotic macroscopic scat cering cross-section of hydrogen $\left(-20.4 \mathrm{~N}^{H}\right.$, where $N^{H}$ is the atom density of hydrogen). The quantity $\mathrm{B}_{\mathrm{av}}{ }^{2}$ is computed to bo

$$
\mathrm{B}_{\mathrm{av}}^{2}=.087981
$$

in a Nelkin model.

Computations of EQ (E) from Eq. (5) were inst performed corresponding to room temperature water; these were compared to computations done by J. Hardy and reported in reference 6 (a larger version of Figure 1 of 
reference 6 was obtalned directly from J. Hardy.) The purpose of this comparison was to verify our use of the corngold asymptotic expanston. J. Hardy, Jr. used seven terms in the expansion, whlle we used five terms. Agreement within.27 was obtained. Calculations for both a plain water case and water polsoned such that the ahsorption cross-section (including the hydrogen absorptıon) was 6 barns per hydrogen atom at $.0253 \mathrm{eV}$ were performed.

Calculations of eptthermal fluxes by the Corngold asymptotic expansion were then performed at reactor operating temperature (more precisely, : $\left.00^{\circ} \mathrm{F}=533^{\circ} \mathrm{K}\right)$. The calculations were for $\mathrm{plain}$ water and for water polsoned with $e \frac{1}{v}$ absorber such that the polson absorption cross-section per hydrogen atom was 6 barns at $.0253 \mathrm{eV}$. The reaults are compared to similar cases at room temperature in Figure 1. The effects of moderator temperature on the eplthermal flux is seen to be falrly small.

Compartson of epithermal fluxes as calculated by the Corngold asymptotic expanston, and by the HAQRR program with the new hydrogen library represinting hydrogen bound in water, are presented in Figure 2 . The same figure shows the fluxes as calculated with the old hydrogen library which corresponds to a free proton at rest model. These comparisons are for the case of plain water; figure 3 gives a similar comparison for the case of water poisoned with a $\frac{1}{v}$ absorber, wh the polson absorption being 6 barns per hydrogen atom at $.0253 \mathrm{eV}$. The fluxes calculated using the new Hatork hydrogen cross-section set agree rather well with the Corngold expanstion fluxes, and represent a contiderable improvement over those calculated with the old HAMIER hydrogen cross-section set.

\section{The Changes to the MAMER Program}

In addition to the changes to the HaMeR epithermal library, it is necessary to mate changes to the HAYYFR program in order that chemical binding effects be taken into account properiy.

The first of these changes is in the subroutine SDRE to ensure that the library values of $\mathrm{E}_{\mathrm{s}}$ and $\vee$ for hydrogen art used in the heterogeneous part 
of the slowing-down calculation. Originally, HAMME treated the slowingdown density due to hydrogen everywhere by the equation

$$
\frac{d q_{H}}{d u}+q_{H}=\Sigma_{s H^{q}},
$$

where $q_{H}$ is the slowing-down density due to hydrogen, $\Sigma_{s H}$ is the scattering cross-section aue $t$, hydrogen, and $\phi$ is the flux per unit lethargy. (See reference 2 for a good description of the HAMER code from the viewpoint of the physical approximations and numerical techniques used.)

HAMER was later modified so that in that part of the above-thermal calculation where the homogeneous slowing-down calculation is done the slowing-down density due to hydrogen is treated by

$$
\gamma_{H} \frac{d q_{H}}{d u}+q_{H}=g_{H^{\Sigma}} s_{H}
$$

The homogeneous slowing-down calculation is done in subroutine SLODON. However, the $S \emptyset R E$ subroutine was left unmodifled.

The primary reason that the changes in the subrout ine SøRE are Important is that the regionwse unltigroup fluxes and slowing-down densittes calculated in the heterogeneous part of the calculation are used in the calculation of the resonance ahsorption probability. In particular (see Eq. (73) of reference 2), the resonance absorption probability for a resonance with resonance integral I is given by

$$
A=1-\exp \left\{-\left(N_{0} I \Phi_{0} v_{0}\right) / \sum_{n} q_{n i} v_{n}\right\}
$$

where $q_{n i}$ is the slowing-down density in the $n$th region at the lower energy IImf of the $i$ th multigroup (before the ith multigroup resonance absorption depletes the slowing-down density), and is due to all isotopes, both hydrogen and heavy elements. The quantity ${ }_{0} v_{0}$ is the Integral of the $i$ th multigroup flux over the regions containing the resonance isotope, and $N_{0}$ is the number density of the resonance absorber averaged over these regions. Consequently, the resonance absorption probability is affected directly by the slowing-down densities and fluxes entering into the heterogeneous part of the above-thermal HAMER calculation. 
In addition to the changes in the SøRE subroutine, changes must be made to the Dancoff factor calculation and the calculation of the collision probability calculations used in the resonince integral calculation for the Pu-240 $1 \mathrm{eV}$ resonance. In point of fact, the changes are made for all resonances which occur in multigroups 53 ard 54 . They are made only on option in the program. The option is used whenrver the 30th element on the batch control card is a 1 , (This sets a flag ICADY equal to unity in the program.)

Betore discussing these changes in detall, it should be pointed out that the older version of HAMPER did not recalculate the Dancof $f$ factor in each multigroup, but rather used the Dancoff iactor calculated in a given multogroup in all succeeding multigroups. Thus the Dancoff factor, if it was calculated at all, was calculaied only once per problem. Whether or not the collision probabllities used in the resonance integral calculation are obtaine $\rfloor$ from a formula using a Dancoff factor or whether they are calculated by the method of cosine currents depends on whether the flux across the lattice cell 1 s suffictently flat (see reference 2 here). If the flux is sufficiently flat, then a parameter KEY is set equal to unity, and the Dancoff factor method is used when the resonance absorber is in the fuel pellet. However, once the parameter XEY is set equal to unty it is not reset in any succeeding multigroup, and moreover the collision probability table is not recalculated in the succeeding multigroup. This had to be changed in order to modify the resonance integral calculation for the Pu-240 $1 \mathrm{eV}$ resonance so that proton chemical binding effects could be taken into account. Changes were therefore made to the subroutine FLuX so that the Dancoff factor would be recalculated in each maltigroup.

In accordance with the method described in references 7 and 8 , the modifications to the Dancoff factor are made by computing the "optical thickness" ¿ar of each reglon external to the fuel pellet stack with

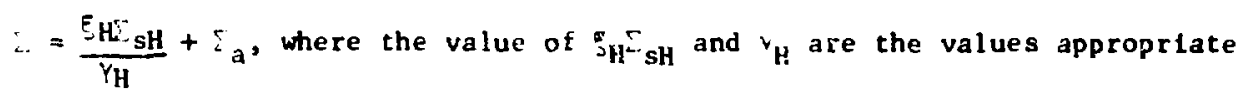
to hydrogen for that group; here $\Delta r$ is the difference between the outer and inner radit for the particular region, and $z_{a}$ is the absorption cross-section 
for the given region. The change is made only if there is a 1 in colum 30 of the batch control card in the input to HAMER. Under these circumstances, it affects all resonances in multigroups 53 and 54 . This change occurs in the subroutine DANC. The change Implicitly assumes that the resonances in question are wide wh thespect to scattering From all isotopes except hydrogen.

As mentioned earlier, if the flur is not sufficientiy flat across the lattice cell the Dancoff factor is not used to calculate the table of collision probabilities used in the resonance integral calculation. Instead a method of cosine currents is used. Here the collision probability calculation was modified by using ${ }_{3} \Sigma_{\mathrm{sH}_{\mathrm{H}}} / \gamma_{\mathrm{H}}$ as the cross-section in each region (except in the region containing the resonance absorber) where previously the quantity $\Sigma_{s}$, the scattering cross-section of all materials, appeared. This change occurs in the subroutine PTBL.

The appendix gives, along with some comments, a Fortran listing of the subroutines which have been changed. This is a preliminary version of the program.

In an early version of the program there were certain indications of errors in the resonance integral calculation performed for the Pu-240 $1 \mathrm{eV}$ resonance. These errors could have been associated with the coarseness of the energy mesh used in the numerical calculation of the resonance integral, or possibly some sort of numerical instability (see reference 12). Later work indicated that the numerical instability problem is not serious, and that the use of a finer mesh in the resonance integral calculation was sufficient. The changes to the subroutine GRID which are required to permit a finer mesh in the resonance integral calculation are included in the appendix.

Concerning limitations on the use of the new program and the new hydrogen library, one should probably use the new library at all times; the use of the ICADY = 1 option (activated by putting a 1 in column 30 of the batch control card) should be used only if the princiaal moderator is hydrogen. 


\section{APPENDIX I}

This appendix gives a Fortran listing of those subroutines whict. have been changed, with some explanatory coments. The new cards added to the source are readily identifiable since they are labelled with the identification "HAMME". The deleted cards are not show, however.

The changes to the subroutine RTBLIB consist in:

(1) Setting the flag ICADY by equating it to ICNTRL (158), which (see LINK 1 of the HAMER program) is equal to the item in column 30 of the batch control card.

(2) Computing the parameter $\frac{\xi_{H i} \text { sH }}{Y_{H}}$ for each mixture, For the Mth mixture this variable is called $\operatorname{CDYC}(M)$. If, In any mixture $M$, the hydrogen concentration is so low that the value of $N^{H} \gamma_{H} E_{H} C s H$ is less than $.0001, \operatorname{CDYC}(H)$ is set equal to zero.

(3) A labelled common block CADY is used to transmit CDYC(M) and ICADY to other subroutines where t'iey are required.

The changes to the subroutine SwRE consist in:

(1) The computation of $Y$ for hydrogen from the ratio of $\left(\mathrm{NY}_{5} \mathrm{~J}_{S}\right)_{H}$ to $\left(\mathrm{NE} \sigma_{\mathrm{S}}\right)_{\mathrm{H}}$.

(2) The kernel $P_{n^{\prime} 1}$ (see eq. (44) of reference 2) is modified to use the library value of $\checkmark$ and $g \Sigma_{s}$ for hydrogen. This kernel expresses the effect of in-group scattering in the equation determining the fluxes in the various regions of the cell. It is denoted by $\operatorname{CRP}(\mathrm{N})$ in the program.

(3) The slowing-down density due to hydrogen (called ETA(N) in the program) is computed with $v$ and $\xi_{5}^{-} s$ for hydrogen used where unity and $I_{s}$ were used previously. The equation is now completely analogous to the equation for $Q(N)$, the nonhydrogenous slowing-down density. 
(4) The source term $\left(s_{n^{\prime}}\right.$ of eq. (48) of reference 2$)$ is iodified also to use the $\gamma$ and $\Sigma_{2} \Sigma_{s}$ for hydrogen. This term is denoted by $S \emptyset R(N)$ in the program.

The source $S_{n^{\prime}}$ and the kernel $P_{n^{\prime}}$ discussed above occur in eq. (40) of refertence 2 ,

$$
F_{n 1}=\sum_{n \cdot} T_{n n^{\prime} 1}\left(P_{n^{\prime} 1} t_{n ' 1}+S_{n \cdot 1}\right)
$$

where $n$ and $n^{\prime}$ are region indices and 1 is a group index. Th1s equation determines the regionwise fluxes.

A change to the subroutine FuX is mide so that the flat flux flag KEY is initialized to zero in each group and $l:$ reset to 1 only if the $f l a t$ flux criterion is met. Certain indices MDdNE(I) are reset to zero in each group. This ensures that the coilision probability tables will be recalculated in ach group.

Changes to PTBL are made. They consist of

(1) Defining IGCDY as IG, the group index. Th1s index is transiltted to DANC through the labelled common block CADY.

(2) The eross-section XT(N) used in regions not contalning the resonance absorber for the collision probability calculation when the method of cosine currerts is used is set equal to CDYC(M) instead of the scattering cross-section for groups 53 and 54 if ICADY $=1$.

Changes to DANC are made, to the calculation of the moderator optical thickness SL, for groups 53 and 54, if ICADY $=1$. In such cases the optical thickness SL is computed with CDYC(M) instead of the scattering cross-section $\operatorname{ssc}(M)$.

Changes to subroutine GRID have been made so that more than 501 mesh points (in lethargy) can be used per resonance. The mesh for all resonances in multigroup 53 is changed to half that used in other multigroups. These changes reduce the mesh error in the treatment of the $1 \mathrm{eV}$ resonance of Pu-240 to an acceptable level (about $1 \%$ ). 
SI:ZOUTINE FLUX(10)

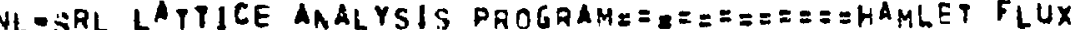

CFLIIX ITERATE OA SPATIAL DISTHIEUTICN OF FLLX

EIFESSICN CNTPLI2UOI:BTPLIO):LNK(10)

COMMON CNTRL

EOUIVALENCE

X(CNYRLI 1), IDBCH), (CNYRLI 2),NCASE),(CNTRL( 3),NFLOG),

1 (CNTRL ( $\left.{ }^{4}\right)$, QTTL(1)), (CNPRLI 13), NTIN), (CNTRL (14), NTOUT),

2(CNTRL ( 29),NTPUN), (CNFHL(16),NCHAIN), (CNTAL 17$), L I A I)$.

3 (CNTRL, 1E). LIR2), (CNTAL( 19), LIBJ). (CNTAL 20):INT1;:

4(CNTRL( 21), INT2):(CNFAL( 22), NSC1), (CNTRL( 23$), L N K(1))$,

$5\left(C N T R_{L}(33), N^{\top} H C S\right):\left(C N T A_{L}(34), N E P C S\right),\left(C N T A L(35), K L 1 B_{1}\right)$,

6(CNTRL ( 3E),KLIB2), (CNYRLI 37),KLIBJ): (CNTAL(124),NCPRN),

7 (CNTRL (130), NCPPY); (CNYAL (141), NTHRA). (CNTRL (142), NTHPT),

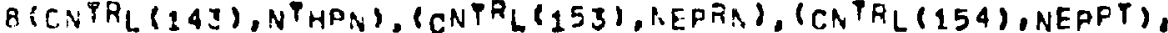

9(CNTRL (15E).NEPPN), (CNPHL(156), NEPSG), (ENTRL (157),NEPAG):

$A(C N T R L(165), N F G F N),(C N P R L(166), N F G P T),(C N T R L(167), N F G P N)$,

B(CNTRL (168), NFGPB), (CNYRL (169), NFG JO): (CNTRL 1177$\left.), N E D^{R} N\right)$,

C (CNTRL $(178), N E D P T), I C N Y A L(179), \Lambda E E F A),(C N T R L(180), N E D N B)$,

D(CI.TRL: 181 ). NEDFW:, (CNYRL $\left(18_{2}\right)$, NECFE), (CNTHL $\left.(183), N E D^{A} X\right)$.

E (CNTRL (184), NFDNU)

DINENSICN LIMP(6OO), HOL $(9), N F T(\angle 0), T H Y(20), H X A S(20)$,

1 IL.H! (2C), ARBP(20), DEGC (20),WSTBA (18), WSTBB(1B),

2CONCTA $(18,10)$, L IMT (10), AIN (20), FAO(20), ROUT (20), VOL(20),

3MTHL(?i), FEGV $\{20), F\{S D(20\}, W O L I[(3,18)$

COMMON LINP

EOUIVLLENCE
IILIMP( 1$)$,

$3(L ! M P(7)$,

4LIMP: 10). IDENY): (LIMF! 2$),$
NRX): (LIMF? 5 ). NGEOM): (LIMF( 8$)$. NXP): (LIMP( 111 .

5 ILIMP( 21$)$ NPT (1)! (LIM'(4).
$A x),(L \operatorname{LP}(3)$ ISOX). (LIAP( 6$):$ AP1B1!. ILIMP!

BSOD), (LIMP( 12). THT(1)), (LIMP( 61$)$ :
FLUX

FLUX

Fiux

FLUX

FLUX

FLUX

FLUX

FLUX

FuX

F) $u x$

Fin $4 x$

FLUX

Fu UX

Fiux

FLUX

fLUX

FLUX

FLUX

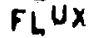

FLUX

FLUX

Fivx

FLUX

FLUX

FLUX.

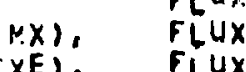

AECT), FiUX

HCL $(1)), \quad$ FLLX

MXAS(1)), FLUX

DEGC(1), FLUX

7(1. IMP:141),WSTBA(1)):(LIMF(159),WSTEB(1)), (LIMP(17):COACTA(1,1)i

8(LIMP(357), LIMP(1):, (LIMF(36)), RIN(1)), (LIMP(3L7): RAD(1))

9(LIMP(407), ROUT(1)): (LIMP(42), VCL(1)), (LIMP(447): MTEL(1)),

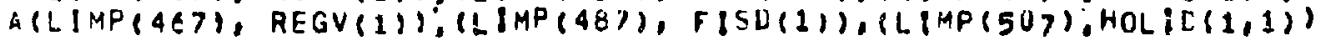

DIMEASICN LOUT(1100):HOLA(9), EAIT(1E,4,5),FO(L $(20,4,5)$ :

$150 r_{3} \times(4,6), F O X(20,4), U_{M H}(20), C T \in C(5), C^{\top} G A(30,5)$

COMYON L.OLT

SIIVALENCE ( LOUT(1),ID), (LCUT(2),HOLA(1)):

1 (LCUT(11):BALT $(1,1,1)),(L C U T(371), F C(L(1,1,1))$,

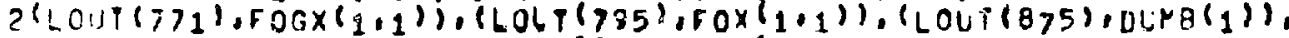

3 (LOJT (895), OTGC(1)), (LOUT $(900)$, CYGA (1))

DIMENDICN ERASE 3500$)$

COMMO:N ERASE

DIMENSICN SSC(10),S1,10),SIGAES(10),SIGIN(10),SIGX(13):C(1C), 1AV(10), SD(10),PROR(25,1U), S(GY $(10,54), G N U(18,54), S j G A(19,54)$, ZNRIG(18),ER(200 ),GMN(200),GNGN(200),GNF(200) :,5PIN(200),

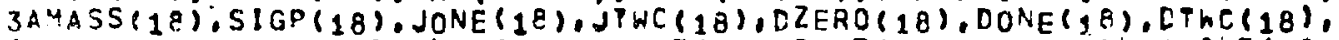
$4 E T A(20), O(20), F S(54) ; F \times(20), L E L(54), F(20,54), H D O N E(18), P(18,54):$ 5PF (18:54),PZ(1001),PZ1(1001),A1(18),EEK(55), TEMP(18), SIGEFY(18). $6 \mathrm{CT}(18,10)$

COMYCN IX, SIGY,GNU,SIGA,P,PF,F, R.EL,FS,SSC,SI,SIGABS,SIGIN,SIGX, IC, AN, SE, PGOG, VRIG,ER;,GHY,GMEM,GFF,SFIN,AMASS, SIGP, JONE, JWC,

\section{$F$ LUX}

FLuX

FLUX

FLUX

FLUX

FLUX

FLUX

FLUX

FLUX

FL.UX

FLUX

FLUX

FinX

Ffux

FivX

FLUX

FLUX

FLUX

FLUX

FLUX

FLUX

FLUX 


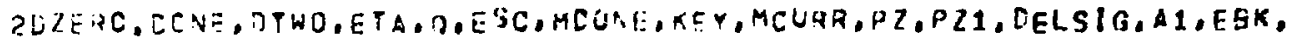
$S T$ THP, SIGETY,NDONE

DIMESSICN DATA (165), DZ(19), GRP( 20$), S O R(20), P(20,20), X^{T}(20), V 1(20)$; IV2(20),V3(20), V4(20), VS(20),CP:20),CN(20), CP2(20), CN2(20), Y(21), 2F $1(20), F 2(2)), 52(20) ; 01(20), 6 C(20), 10(20), 11(20)$

EQLIV $\triangle$ LENCE (ERASF(1),DATA(1),D2(1),GRP(1)),

1(ERASE (21), SOR(1)), (ERASE(41),T(1)), (ERASE (4A1),XT (1)): ? (EAASE $(461), V 1(1))$, IERASE $\left.\left(4 \theta_{1}\right), V 2(1)\right),(E R A S E(501), V 3(1))$ 3(ERASE(S21),VA(1)), (ERASE (541),V5(1)), (ERASE (561), FP(1)): 4 (ERASF(E81),CV(1)): (ERASE $(601),(P 2(1)),(E R A S E(621), C N 2(1))$, $5\left(E_{R A S E}(641), Y(1)\right),\left(E_{R}^{A} S^{A}(41), F_{1}(1)\right),\left(E_{R A S E}(461), F_{2}(1)\right)$. 6(ERASE $(481), 52(1)),\left(E^{R} S_{E}(501), C_{1}(1)\right),(E R A S E(481), 00(1))$, 7 (ERAJE(461), TO(1)), (ERASE 441$\left.), T_{1}(1)\right)$

EDUIVALENEE (FX(1),FISD(1)), TCT(1),CONCTA(1), (LIPZ,NLIEZ) COMMCN ALIB

$\operatorname{TCNT}=0$

$\triangle N O R M=0$.

DOSBSON=1,NX

$X N O R M=X A O H M+S O R(N)=\operatorname{VOL}(N)$

$M=M T \equiv L(\Lambda)$

$9998 \mathrm{CO}^{\mathrm{N}} \mathrm{T}$ ING

$Q 1(V)=(S S C(M)-S I(M)+S I G I N(M)+S I C A E S(M)=G R P(N))+V O L(N)$

1

$f F(10-1)=1,5$

$\operatorname{lit} \mu_{i} x=0$

Fi: $X=1$.

$|G|=1$

$I G R=1$

EPSF $=0.1$

ITMAX $=100$

$E P S=1, E-3$

Lo $3 \quad N=1, \wedge x$

$3 \quad F I(N)=S C F(N)$

Go io 20

$5 \quad D O 6 \quad v=1,4 x$

$6 \quad F 1(N)=F(N, 1 G=1)$

20 RNORY $=0,0$

DO $30 \quad i=1, N X$

30 RNDON=FAOFM+OI(N) F F (N)

RNORN $=$ X OFM/RNORM

DO $40 \quad I=1, N X$

$F_{1}(N)=F 1(R)+R N O R M$

$S_{2}(N)=S C R(N)+F I(N), G R^{P}(N)$

$\therefore$ CONTINLE

45

RNORM $=0.0$

CO $53 \quad A=1, N X$

$F 2(v)=0,0$

DO $50 \mu=1, N^{X}$

$D L M M Y=C L M N Y$

$+?(N)=F 2(M)+T(N, M) \cdot S 2(M)$

$53 \quad F=O R:=A R O R M+01(N)+F ?(N)$

$P_{N O R}=X_{N} O^{F_{M}} / R_{\text {VOR }} R_{M}$

55 CO $55 \quad=1, N X$

$F 2(N)=F z(A) \cdot R$ NORM

LO $60 \quad I=1, N^{X}$

DUM MY $=$ CLM*Y

Flux

FLux

$F$ LUX

FLUX

FLUX

Fiux

FLUX

FLUX

FiUX

Fiux

F LUX

$f L U X$

FLUX

fLux

FLUX

FLux

Fux

FLUX

FLUX

FLux

Fiux

Fiux

FLUX

Ffux

FiUx

Fiux

FLUX

Fiux

FLUX

FLUX

FLUX

FLUX

rivix

FLUX

fLuX

FLuX

FLUX

FLUX

Fivx

fiux

FLUX

FLUX

FinX

FLuX

Flux

FLux

FLux

FLuX

FLUX

FLUX

FLuX

FLUX

fLUX

FL UX

fLuX 
IF(ABS( $(F I(N)-F 2(Y)), F 2(V))-E F S) \quad 00.00,70$

:O CONTISLE

GO 1090

$70 D O 80 \quad A=1, N X$

$S 2(N)=S C R(N) \bullet F 2(N) \cdot G_{R} P(N)$

$F 1(N)=F 2(N)$

BO CONTINULE

$I T C N T=\mid T C N T+1$

IF IIICAT-ITMAX\} 45,90,90

90 IT IITCAT-ITMXI92,92,91

$91 T^{\top} M=I^{\top} C N^{\top}$

$I G I=10$

92 IF (ABS (FNCRM-1,)-ABS (RMX-1,)) $94,94,93$

93 RMX = RNCFIM

$1 G^{R}=16$

94 CONTINUE

rolod:1=1, AX

$100 ;(N, I G)=F Z(N)$

$K E Y=0$

$105 \quad$ If $(N x+1) \quad 125,125,110$

119 D0120 $=2,4 x$

IF (ABS(F $2(N) / F 2(1)-1).-E P S F) 120.120 .130$

120 CONTINUE

$125 \quad K E Y=1$

130 DO140 $1=1,15 \cap x$

SIGEFY $(1)=0$.

140 MDONE $(\ell)=0$

MCURR $=0$

NDGNE $=0$

FLUX

FLUX

$F L U X$

FLUX

FLUX

Fiux

flux

FLUX

FLUX

REWINDNSCI

$150 \quad$ IF (54.16)152,151,152

151 WRITEINTOLT.15O)ITMX: IGI, FMX, IGK

16, FORMATIS9ROMAXIMUM ITERATIOAS ON THE FLUX SHAPE IN ANY MACRO GROL 1P $=$ \$5, EH GROUP I2/39H MAXINUN FEMOFMALIZATION IN ANY GROUP = $1 F 9,5,6 H$ G $=$ OUP $(2)$

152 RETURN

FLUXX

FLuX

FLUX

Fiux

FLux

FLUX

FLUX

FLUX

HAMMER

FLUX

fiux

FLUX

FLuX

FLUX

FLUX

Flux

fivx

FLUX

FLUX

FLUX

FLUX

FLUX

END

FLUX

FLUX

FLux

FLUX

FLUX

$\ln$

SIGVED OVER THE LEOP BEGINNINU AP LIVE 92

SIGNED OVER THE LCOP BERINNING AT LIVE 95

SIGVEO OVER THE LCOP BEGINN?NG AT LIAE $10 \mathrm{C}$

SIGNED OVER THE LCOP BEGINNING AT LiNF 107 
CRTHLIN

SURRUUTlint RTPLIUIfl,

UJMENSIUY CNTRL (200), BTTL19), LNK(10)

CIMMUA CNTRL

ETUIVALENCE

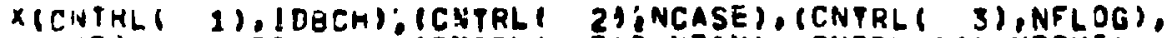
1 (CITRL (48,BTTL(1)):(CNTRL 13I: NTIN), (CNFRL (14), NTOUT). 2(CNTKL( 15), NTPUN): (CUTRL (16), NCHAIN), (CNFRL( 17),L181),

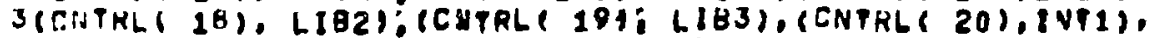
4(CNTKLI 21). INT2): (CNTALE 224: NSC1), (CNTRL( 23),LNK(1)).

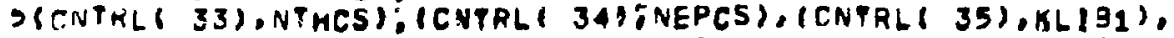

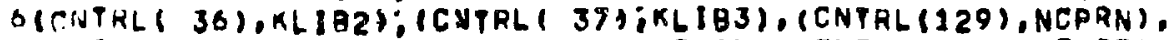
7 (CNTKL' 130 ), NCPPT): (CVTRL (141);NTHAN), (CNTRL (142),NTHOT). H (CNTHL (143), NTHON): (CNPRL (153) NNEPRN), (CNFRL (154),NEPPT). Y (CNTYL (155), NEPQN), (CYIRL (156) NEPSG), (CNTRL (157), NEPAG). A (ENTRL (165): NFGRN): (CUTRL(166):NFGPT): (CNTAL(167):NFGDN): H(CVTHL (16H), NF GPG): (CVTRL (169): NF GJO) , (CNTRL (177), NEDRN). C (CNTHL (178), NEDDT): (CVTAL (1991 :AEDN), (CNPRL (180),NEDVE). I) (CNTRL (181), NEDFW); ( MTRL (1R2) NEURB), (CNTRL (183),NEDAX), E(CNTRL (1\&4). NEUVU)

DIMENSIUN LIMP(SOD), HOL $(9), N P T(20), T H T(20), M X A S(20)$. ILLI(20), NRAP(20),DEGS(20),WSTUA(18), WSTGB(18), PCONETA $(18,10), L(M T: 10)$, RIN(20), KAU $(20)$, RUUT $(20), V O L(20)$. SMTBL (20), REliV(20),FISD(PO), HOLID(3,18)

COMYUN LIMF ETUIVALENCE IYLIMP! II, ?(L) पस 4). S(LI"4) 7$)$.

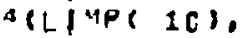
(LINAT 21), NPT(1)I, LIMP: 41).

IDEVTI, ILIMP| NZXI.ILIMPI NGEJMI, ILIMPI

21. 51. 81.

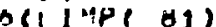
NPT (1): (LIMPF I (LIUP(141), WSTUA(1) , (LIMP(159),WS S(LIMP(357), LIMT(I)), (LIMP (367). 9(L) MP (407). ROUT(1) I, (LIMP(427).

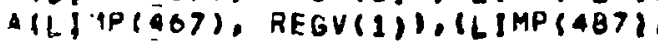
UIMENSIUN

$1+C . G \times(4,0), 50$

COMMON LOUT

EJUIVALEVCE

I (LUJI(11), BALT (1,1,1) , (LOUT (371),FOIL $(1,1,9))$,

?(LO:17 $(771), F O G \times(1,1),(L O U P(795), F O \times(1,1)),(L O U T(975), 0 U M B(1)) ;$

$3(10.1 \overline{1}(095), 0$ TGC(1)), (LOIIT(900);0TGA(1))

UIMEÑSION ER.ASE 350 O

CDMUUV ERASE

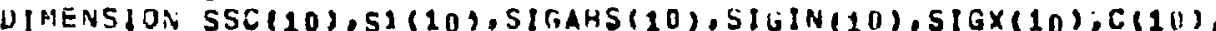

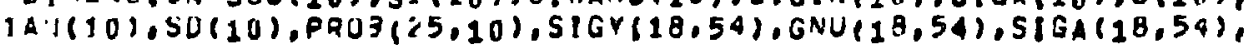
PNR II(10), ER(200), GMN(200),6MGM(200), GMF(200) 1,5PIN(200), 3A4ASS(16),SIGP(16): JOVE(18), JTNO(18), DZEHO(18),ODNE(13).0T:0(18), UETA $(2 \pi), \partial(20), F S(54), F \times(20), D E L(54), F(20,54), M D O N E(18) ; P(10,54)$, SPF(18,S4),PZ(1001);PZ1(1001),A2(18),EBK(55),TEMP(18), SIGEFY(18), $3 \operatorname{CT}(16,10)$

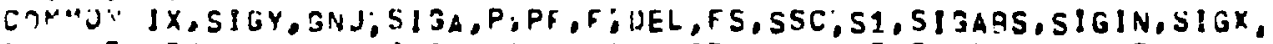
IC, ". SU.PHOH, NHIG.EF, GMN,GMRN, GMF,SPIN, AMASS,SIGP,JJNE, JTWU. ?UTE+O, LONE, TTWU,ETA,O.ESC, KDONE,KEY, MCURR,PZ,PZI, OELSIG, $1, E B K$,
RTHL!

RTHLI

RTBLI

RTGL!

RTELI

RTBLI

RTALI

RTHLI

RTHLI

RTBLI

RTBLI

คTBL!

RTEL

RTBLI

RTEL

RTHLI

RTHLI

RTHL I

RTHLI

RTISLI

RTHI I

ATALI

RTKLI

RTBLI

RTAL:

RTHL

RTHL

RTHL

RTHL

RTHL

RTEL

RTHL

RTBL

RTHL

RTHL

RTAL

RTHL

RTHL

RTEL

RTHL

RTEL

RTBL

RTHL

RTHL

RTHL

HTEL

RTRL

RTEL

RTHL

RTHL

RTHL

HTRL

RTHL

ATHL 
SIEHW. SIGEHY ONDUVE

UIHENSIUN UATA(165),D2(18),GRP(20),50R(20),T(70,20),XT(20), V1) 20$),$ $1 \vee>(20), V 3(20), V 4(20), V 5(20), C P(20), C N(20), C P 2(20), C V 2(29), V(21)$, 2F+120),F $2(20), 52(20), 01(20), 00(20), 70(20) ; 71(20)$

EDUIVALENCE (ERASE(1).DATA(1).02(1),GRP(1)).

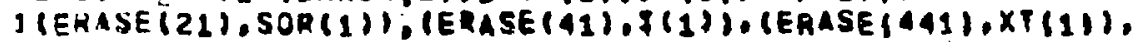
2(ERASE (401), V1(1)); (EAASE (481); V2 (1)), (ERASE $(501), V 311)$,

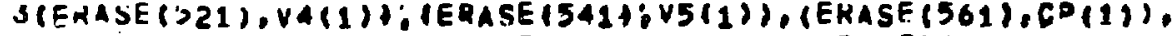
A (ERASE (SU1), CN(1));:IEAASE(601);CP2(1)i, (ERASE(621), CN2(1)),

S(EKASE (041), Y(1)), (ERASE (441), I $1(1)$ ), (ERASE $(461), F 2(1))$. O(EHASE (481), S2(1)): IERASE (501): W1 11$)$, (ERASE $(481), 00(1))$,

T(EKASE (401), TO(1)): (ERASE $(441)$ T T1(1))

EJUIVALENCE (FX(1); ISD(1), (CF(1),CONCTA(1)), (LIB2,NL1B2) COM:AOV NL IO

DIMENS IUN THFLX(630), ANH(10), SUH(10), S1H(10)

COMMUN THFLX, ANH, SOH, S $1^{H}$

DIMENSIUNLOCRI20)

EJUIVALENCE (ERAST(3441),LOCR(1))

COMMUVICREG $2 / 0 S_{0}(16,54), 0 S_{1}(19 ; 54), 0 \times S_{0}(18,54)$

DIMENSION AHSSIG(18), FISSIGI18), GNUSIG(18), SGSIS(54)

EJUIVALENCE

1 (5GSIG(1).ABSSIG(1)),(SGSIG(19),FISSIG(1)), (SGSIG(3),GNUSIG(1)) COMMON/CADY/CDYCI 101 , IGCDY, ICADי

DPMENSIUN ICNTHLI2OUI)

EJUIVALENCE (ICVTZLII), CNTRL(1):

ICAIY = \CNTRL (15R)

IF(IL.EU,1) WRITE(NTOJT,1111) ICADY

1111 FOHMATS* C ICAOYA*II)

I $S U X=I S U X+1 S O X_{C}$

Uns $I=1: 34$ t

$5 \operatorname{ssc}(1)=0$. Un $01=1.30$

6 ANH $(1)=0.0$

IFOUNDEO

NRED

NLI:

10 NRE:I=NKED+1

REAU(LIUZ)IFL,WOX,NIN,NR , DATA(I),IEI,IFL)

IF NUX)14C,140.2C

? Uา110I=1.ISUX

IF (AUX:NE. WSTEA(!)) G9 TO 110

30 SILY(I:IG)=UATA:b)

GiJU(J, J (G) =LATA (9)

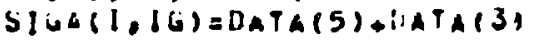

FISSISIJ) $=$ SIGY(I,IG)

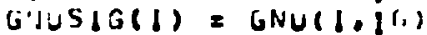

ATSSI3(1) = SIGA $(1,16)$

USA $(1,1 G)=U A T A(?)+D A T A(4)$

$0,1(1,1 G)=[A T A(7)$

Oxbi $(1,10)=$ I)

$\because>1,(1)=\mathrm{y}$ :

f $5(: 2) \leqslant 1,34,31$

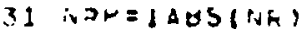

$L: C+(1)=$.

Lาง $1:=1$. .4 $=$

RTHLIE

RTELIO

RTALIO

RTHLIB

RTBLIB

RTRLIB

RTHLIB

RTBLIE

RTHLIB

RTBLIE

RTHLIB

RTULIO

RTBLIE

RTHLIB

RTHLIH

RTHLIB

RTHLIB

RTHLIB

RTHLIB

RTHLIH

RTHLIB

RTBL I B

HAMMER

HAMHEK

HAMMEH

HAMMER

HAMMEA

HAMMER

RTHLIH

RTHLIB

RTHLIG

RTAL16

RTHLIH

ATHLIB

HTHLIU

RTHLIR

RTHLIE

RTELIE

RTBLIO

RTHLIE

PTHLIE

RTHLIK

ATHL19

กTEL IE

HFin

Artita

คTAL

คT HLIE

ATHL IE

คTHL 16

ATPLI

ATHL

A) 4 16

ค) 416

ATVLI4 


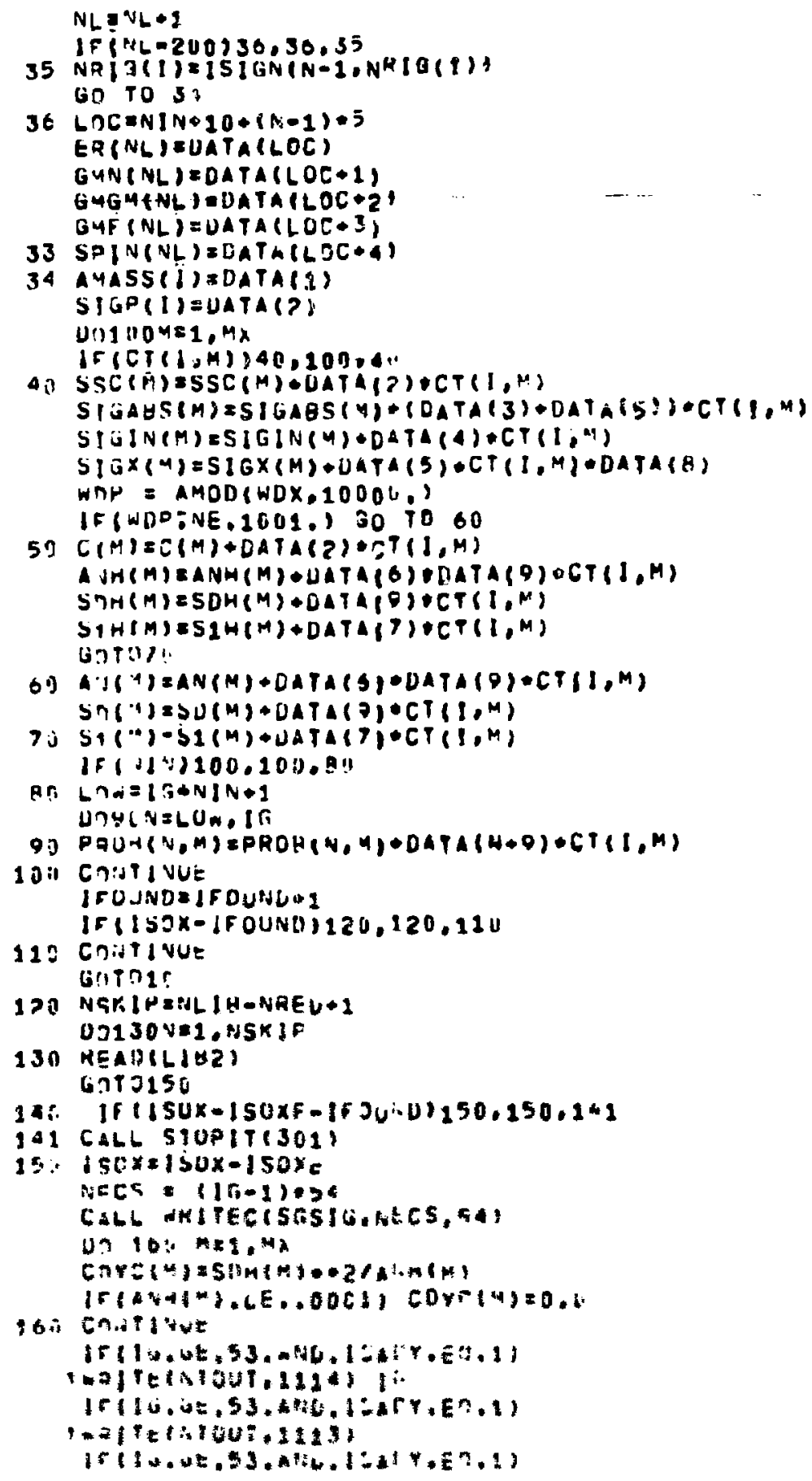

RTHLIE RTHLIE RTALIE HTHLIE RTELIL RTBLII RTELIf RTELIE RTHLIt ATHLII RTELII ATBLII PTHLII RTHLII RTALII RTคI! RTHLII RTHLI RTELI RTHLI RTBL! RTELI RTHLI RTGLI RTELI RTALI RTHLI RTHLI RT'LLI RTELI RTHLI RTHLI ATELI RTHLI RTRLI RTALI RTELI ATRLI RTELI RTELI कTHLI ATHLS RTALI RTHLI DTALI RTHLI HA MMF WAMHE MAMME WAMES MAMMI MAMMI MAMHI MAMAI HAMMI 
FOUTINE RTELIH

CDC 6000 TIN V3.003261 UPTE2 11/20/

INRITE(NTOUT,211?) (CDYC(MI,MET:MX)

1112 FHOAT(DESO.B)

1114 Fia

1113 OOMATP CUYC(M), Yzi, $y x+1$

RETUK!:

ES.

HAMM

HAMM

HAMM

HAMM

RTEL

RTHL 
CSORE

SUERCUTIY: SORE(1r,

DIMESSIEY CNTRL(200):BTTL(9),LA*(10)

COMMCA C:NTRL

EOUIVALENCE

X(CNTRL( 1 ).INATH), (CNTZLI 2),RCASE),(CNTRLI 3),NFLOG),

1 (CNTRL (4), BTYL(1)), (CNTAL 13 ). NFIA). (CATRL (14), NTOUT).

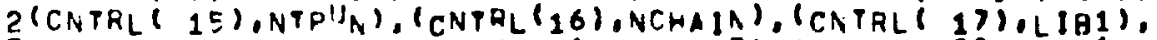
3 (CNTRL ( IE), LIB2), (CNYRLP 14), LIAJ), (CNTRL( 20),INT1). 4 (CNTHL 21 ), INT2), (CNTRL 22$)$, NSE1), (CNIRL 23 ).(NK(1)), $5\left(C N T R_{L}(33), N^{\top} H C S\right),\left(C^{N} T R_{L}(34), N E P C S\right),\left(C N P R_{L}(35), K L I B_{1}\right)$, 6(CATAL ( 3E),KLIA2), \{CNTRLI 37),KL 163), (CNTAL (125), NCPRN), 7 (CNTRL (130), NCPPT); (CNTAL (141), NTRRA), (CNIRL (142) NNTHPT): $\left.8(C N T F L(143), N T H P N), C_{C N T R L}(153), A E P R A\right)$ (CNTRL 154$\left.), N E P P Y\right)$, 9 (CNYRL (155), NEPPN), (CNTHL (156), NEPSC), (CNPAL (15) ) NEPAG). A (CNTRL(165), NFGRN), (CNTHL (166), KF CPT), C CNTRL (167), NFGPN).

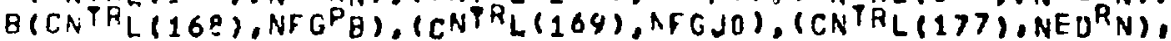
C (CNTRL (17E), NEDPY). (CNTHL (179), NECPA), (CNTRL (180), NEDNB). D (CNTFL (161), NEDFW), (CNTRL (182), NEtRE), (CNTRL (183), NEDAX), E (CN $\left.{ }^{T R}(18 d), N E D H U\right)$

DIMENSICN LIMP $(600), H O L(9), A H T(20)$, THT $(20)$, MXAS(20),

$1\left[L H !(20), R R^{A P}(20), D F G^{C}(20), W T^{B A}(18), W S T^{B B}(18)\right.$,

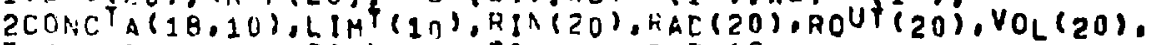

3MTBL(20), EEGVI20); ISD(20), HOLIC $(3,18)$

COMMCN LINP

EOUIVALENCE

1 (LIMP! 1).

2(LIMP: 4),

$3(L I M P(7)$.

IDENTI: (LIMF! 2$),$ ARX): (LIMFE 5 ), NISEOM): (LIMF( E), NXP): (LIMF( 11$)$.

$4(L ! M P(10)$.

5 (LIMP( 21).

7 (LIMP (141), WSTBA (1)): (LIMF (153),W

B (LIMP(357), LIMT(1)): (LIMF 367$)$,

9(LIMP(407), ROUT(1)):(L] $4 F(42))$. A (LJMP(4C)), REGV(1)):(LIMF(48)), FISD(1)), (LIMP(507):HOLID(1.1)

DIMENSICN LOUT(110J): HOLA(9), HALT(18,4.5), FOIL $120.4,5)$ :

$\left.1 F O G \times(4,6), F O \times(20,4), D^{11}, 5(20), C T C C(5), 0^{\top} G A(3), 6\right)$

COMNCN LOLT

EOUIVALENCE

LOUT(1), ID), (LCUT(2),HOLA(1)):

1 (LOUT(1), BAL $(1,1,1),\left(L C U Y(3)_{1}\right), F C\{L(1,1,1)$ )

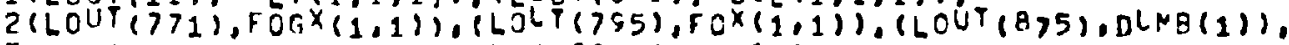

3 (LOUT(895), OTGC(1)), (LOUT(900), T.TCA(1))

DIMENSICN ERASE (3500)

COMMON ERASE

DIMENSICN S5C(10), $S^{1}, 101,5 I G A E S(10), S I G I N(10), S(G \times(1)), C(10)$,

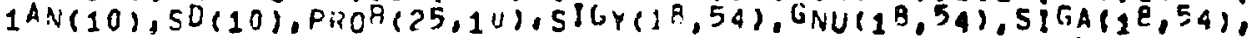
2NRIG(18),ER(200 ), GMN(201) ),GNGN(200) ),GNF(200 1:5PIA120R 1.

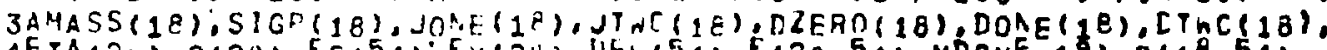
4ETA $(26), 0(20), F 5(54) ; F \times(2 U), U E L(54), F(20,54), M D O N E(18), F(1 E, 54)$. 5PF (18,54);PZ(1001),PZ2(1NU1), A1 (29),EGK(55), TEMP(1B):SICEFY (1E). 6CT $(18,10)$

COMMON IX,SIGY,GNU,SIGA,P,PF,F, TEL,FS,SSC, S1, SIGABS,SIGIA,SIGX, 1C, AN, SE,PFOG, NAIG,ER, GMY, EHCM, GNF, SPIA, AMASS, SIGP, JONE, ¿THC,

2DZERO, CCNE, DTWO,ETA, O, ESC, MLUAE,KEY, MCURA,PZ, PZ1, DELSIG, A, EBK,
SOHE

SCRE

SCAE

SOAE

SOHE

SOHE

$5 \mathrm{O}^{2} \mathrm{E}$

SORE

SORE

SORE

SORE

$S_{0}{ }^{R}$

SOHE

SORE

$S^{A E}$

SORE

SORE

SOAE

SORE

SORE

SORE

SORE

SORE

SoRE

SORE

SORE

SORE

SORE

SOAE

SOAE

SORE

SORE

SORE

SORE

SORE

SoRE

SOHE

SOAE

SOHE

SORE

SOHE

SOHE

SORE

SCHE

SOHE

SOH:

SOHE

SOHE

SOAE

SOHE 
3T $=M P, S:$ SEFY, NONE

SORE

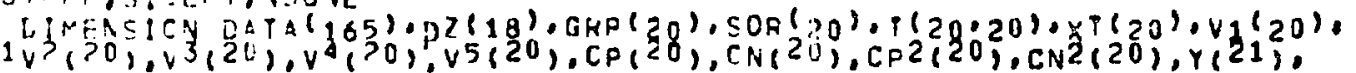

2F $1(20), F_{2}(20), 52(20): 01(20), 0 C(20), T 0(20), T 1(20)$

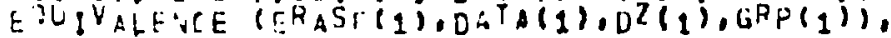

1 (T2ASE(E1), SOR(1)), (ERASE (41), T(1)), (ERASE $\left.441,: X^{\top}(1)\right)$,

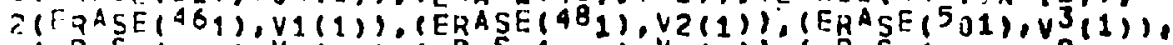

$\left.3\left(C^{R} A S_{E}(521), V_{4}(1)\right),\left(E_{A}^{R_{A}} S_{E}(54), v_{5}(1)\right), V_{E}{ }_{A} S_{E}(561), C^{P}(1)\right)$

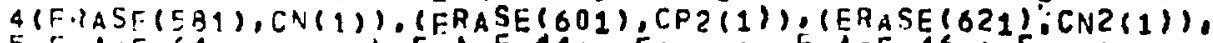

$\left.5\left(F \gamma^{A} S^{C}(E 41), Y(1)\right) ; F_{R}^{A} S^{E}\{41), F_{1}(1)\right),\left(E_{R A} S^{A}\left(46_{1}\right), F_{2}(1)\right)$,

G(FRASE(481),S2(1)), (ERASE(501), 61(1)), (ERASE(481),0011)i,

7 (ERASE (461), TO(1)), (FRASE (441), 11(1))

EOUIVALENCE (FX(1),F ISD (1)), (CT(1), CONCTA(1)), (LIB2,NLIE2)

COMMON ALIB

COMNCN THFLX(630): ANH(1"), SCH(111),S1H(10)

DIMFISICN AMH(10), AMBH(1n),SDH1(10)

DIMENSICN
COMMCN/[AVE/RXRFM(20:3)

DINEASICN FSUM(20), ENNP(2U)

IF $(1 G-1) 1: 10,30$

$10 \quad A=0$

Do $11_{N=1, A X}$

$11 A=A+F X(N) \cdot V O L(N)$

If (A) 12,12,14

$12 t=0$

$\operatorname{Cos} 3_{A}=1, N X$

$13 A=A \div V C_{L}(N)$

D) $1 \& A:=1, A X$

$18 F X(N)=1,14$

GOTC16

14 no15v $=1,1 x$

$15 \quad F \times(A)=f \times(N) / A$

16 nC $20 y=1, x$

AME $(10), A M(10), C_{1}(10), S_{D 1}(10)$

SORE

SOHE

SOHE

$S_{O R}{ }^{R}$

SOHE

SOHE

$S_{O P E}$

SORE

SORE

SORE

SORE

SORE

SCRE

HAMMER

HAMMER

SORE

SCRE

SOHE

$S_{O}{ }^{\mathrm{H} E}$

SORE

SORE

SORE

$S_{O^{R}}$

SORE

SORE

SORE

SORE

SOHE

SORE

SORE

SORE

$O(A)=0$.

$E T A(A)=0$.

$502(N)=F S(1) \cdot F \times(N)$

SOHE

SOHE

$M=\square T B L(\Lambda)$

IF(SD(N)),100,101,100

SORE

SORE

SORE

SORE

$1 \cup 1 \sin (\mu)=0,0$

GO PC QD?

$100 A: H(M)=A V(M) \cap B D(M)$

4 C 5 CDNTI:LE

(F) $(5[\mu(N)) 400,40: .400$

411 $A \operatorname{arm}(\omega)=0.0$

$r_{3} O$ iC $1 \mathrm{C}^{2}$

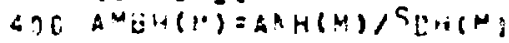

$182[1(M)=C(M)$

$\sin (x)=\sin (\omega)$

$\operatorname{sn} l(x)=e^{2}(y)$

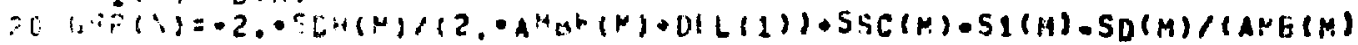

1 - $):(1) / 2,1, f 70^{n}(1, \mu)$

L $1011, i=1.4 x$

$550(5)=0.0$

$1011 E(9)=019=0,0$

FF TUin

SOHE

HAMMER

SOAE

HAMMER

HAMHER

HAMPER

HAMFER

HAMMER

SORE

HAMMER

$S_{O}{ }^{A}$

HAAMER

HAMPER

SOHE

SOAE

SOAE

$S_{S O R}$ 
$30 D O 4 O A=1, N X$

SORE

$M=M P Q_{L}(A)$

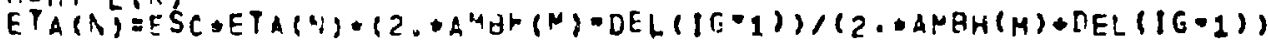

$1 * 2, *[E L(I C-1) * S B H 1(M) * F(N], G \cdot 1) /(2, * A N H H(M)+[E L(; G-1))$

$O(N)=E S C+C(N)+\{2, * A M B(M)-[E L(\mid G-1)\} /(2,+A M B(N)+D E L(\{G=1))$

$1+2, * C E L(I E-1) \cdot 5 n_{1}(M)$ of $(N, I G-1) /(2, \bullet A M G(M)+D E L(1 G-1))$

(F) (IG,EG.55) GO 1040

IF (SD $(M)) Z 00,201,200$

$201 A M(M)=0.0$

GO TC 302

$200 \quad A M(M)=A R(M) / S D(M)$

302 CONTINUE

IF(SDH(F)) $300,301.300$

301 AMH $(M)=0,0$

SO TC 202

$300 A M H(M)=A N H(M) / S D H(M)$

$2 n 2 \operatorname{GRP}(N)=S S C(M)-S 1(M)-2 .+S D F(M) /(\hat{E}, * A M H(M)+D E L(I G))-S D(M) /(A M(M) *$ 1 DE $(f G) / 2$,

$S_{O R}(N)=E Y A(N) \cdot 2, /(2, * A M H(M) * D E L(\mid G)) \bullet G(N) /(A M(M) \bullet D E L() G) / 2,1$

$1+F S([G) \div F \times(N)$

40 CONTIVLE

IF (IG.LT,11) GO IO 1001

IF (IG,GT, 25$)$ GO TO 1002

DO $1003 N=1, N X$

$r=M T_{B L}(\mathrm{~N})$

DO $1004 J=1,10$

ENNP(N) = ENNP(N) $\operatorname{PROG}(,, N) * F(N, J) \cdot C E L(J)$

IF \{ G, NE,11\} GO TO 1004

FSUM(N) $=$ FSUM(N) $+F(N, J)+[E L(\infty)$

1004 CONTINUE

IF (IG,NE, 11) GO TO 1003

$\operatorname{RXREM}(N, 1)=(O(N)+E T A(N)) / F S L M(N)$

1003 CONTINLE

GO TO 1001

$1002 \operatorname{IF}(1 G, G T, E 6) 60 \quad 701005$

DO $100^{6} N=1, N X$

1006 RXREM $(\Lambda, 1)=\operatorname{RXREM}(N, 1) \cdot \operatorname{ENN}^{P}(A) / F S U N(N)$

DO $1012 \mathrm{~N}=1 . \mathrm{NX}$

$\operatorname{SUM}(N)=0,0$

DO $1007 j=11.25$

1007 FSUM(N) = FSUM(N) "F(N,J).CEL(N)

1012 RXREM $(N, 2 S=(O(N) \bullet E Y A(N)) / F S L M(N)$

1005 IF(IG.EG.55) GO YO 1008

GO TO 100 :

1008 DO $1005 N=1, N K$

F SUM(N) $=0.0$

DO $1010 \mathrm{~J}=26,54$

$1010 F \operatorname{SUM}(N)=F \operatorname{SUM}(N) \cdot F(N, J)+D E L(D)$

1009 RXKEM $(A, 3)=\left(O(N) \circ F_{T} A(N)\right) / F S L M(N)$ $G^{\circ}$ IC 80

1001 CONTINLE

DOSOM $=1, A X$

$A_{M} B(M)=A M(M)$

$C 1(M)=C(M)$

$\triangle M B H(M)=A^{M} H(M)$

SORE

HAMNER

HAMMER

SOHE

SOAE

SCRE

SOHE

SORE

HAMMER

SORE

HAMMER

HAMMER

MAMMER

HAMYER

HAMMER

HAMKER

HA HFER

HATMER

HAMMER

SOHE

$S_{O R E}$

SOHE

SOHE

SORE

SORE

SORE

SOHE

SORE

SORE

$S C^{A E}$

SOBE

SOHE

$S_{O}{ }^{P_{E}}$

SOLE

SORE

SORE

SORE

SORE

$S_{D} R_{E}$

SORE

SOHE

SORE

SORE

SORE

SORE

$S_{D^{R}}{ }^{R_{E}}$

SOHE

SORE

SOHE

SOHE

SORE

SORE

SORE

HAMTER 
FTA V3.0.PZEL CFY $=2 \quad 08 / 27 / 74^{Y}$

$S \cap H 1(M)=S[H(M)$

$50 S D_{1}(\mu)=S D(M)$.

HAMMER

SORE

If $(1 G-25) \leq 0,00, R B$

oo $131=13-1$

$D^{0} 70:=1, N^{x}$

$M=M T B L(A)$

$G R P(N)=C R F(V)+P R O B(I G, M)$

SORE

DO70 $=1, ! \& 1$

70 $S^{2}(A)=S^{R}(N)+P R O B(1 ; M) * F(N, I) / C E L(I G) * D E L(I)$

AD RETURN

END

SORE

$S O R_{E}$

SORE

SORE

SORE

$S_{0} R_{E}$

SORE

SORE

:S MADE GY THE CFTIMIZER

IARIANT RLIST REMCVED FROM THE LOCP STAFIINE AT LINE a

ION

IIGNED OVER THE LCOP PEEINNING AT LINE TI

IGINED OVER THE LCOP DEGINNING AT LINE 81

¿GGNEN OVER YHE LCOP PEGINNING AT LINE 86 
SHGYUU! (NE PTHL(13,j)

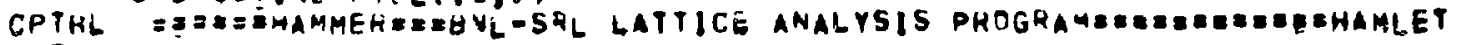
CPTHL

DIMENSION CNTRL $12001,0 T$ OL19),LNK(10)

CIMAUA CNTRL

E JUIVALENCE

X(CITHLI 1).IDGCH), (CVTRLI 24:NCASE),(CNTRLI 3).NFLOG).

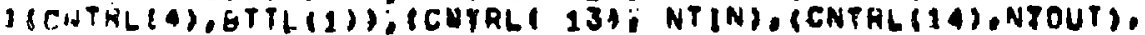

2(CNTKL 15 ).NTPUN), (CUTRLI16),NCHAIN), (CNTRLI 17).LIB1).

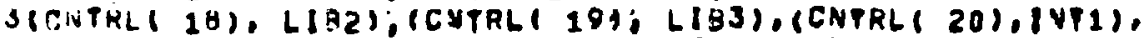

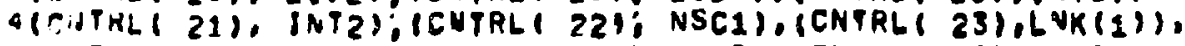

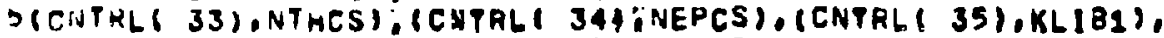

O(CNPRLI 36),KLI32): (CVTRL 37);KLIB3), (CNPRL(129),NEPRN),

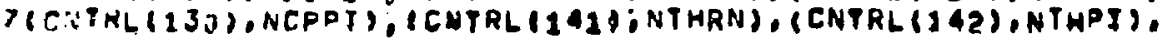

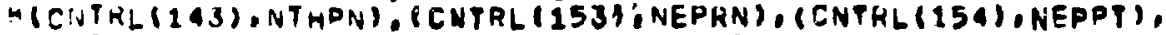

$\checkmark(C, 4$ PL (155), NEPPN): (CYYRL (1561; NEPSG), CCNTRL (157),NEPAG),

a (CVTHL (165), NFGQN), (CNTRL1166);NFGPT), (CNFRL (167),NFGDN),

4 (CNTKL (168).NFGPB): (CNYRL11691:NFGJO), (CNTRL(177),NEDAN).

U(CITRL(178), NEUPT), (CVTRL (2794; NEUPN), (CNYRL (180), NEDVE).

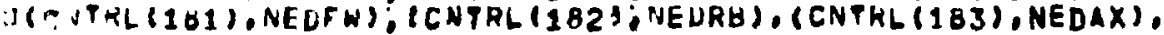

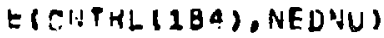

UIMENSJUN LIMP(OOO),H9L $191, N P T(20), T H T(20), 4 \times A 5(20)$.

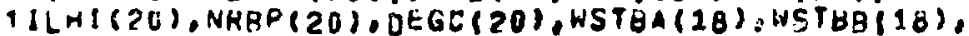

ZCOISIA(1B,10),LIMT(10), RIN(20),HAU(20),RUUT(20),VOL(20).

SMTBL(20),REGVP 20$), F I S D(20), H O L I D(3.18)$

CกMYON LIMP

ETUIVALENCE

$1(L)$ YP 1$)$

¿ाl

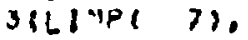

- (L)

IDEVYI, ILIMPI 2$\}$. NRXI. ILIMPI 51 . NGEJMI, ILIMP( 8$\}$. NXPI, ILIMP! 11$\}$.

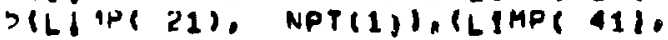

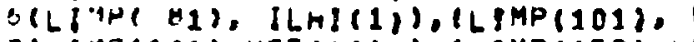

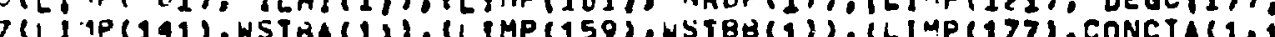

(LIMP(SS7), LIMT(1)), (LIMP(367), AIN(2)), (LIMP(387), RADIJ)).

Y(LIMP(4L)). KOUT(1)), ILIMP(427), VOL(1)), (LIMP(447), MTUL(1)),

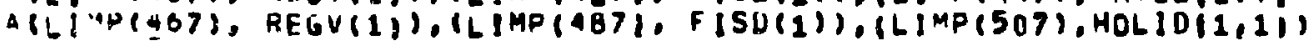

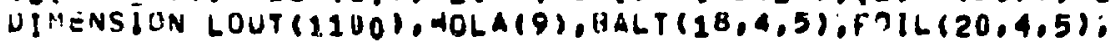

$1+53 \times(4,0), F 0 \times(20,4)$, DUMA $(20), 076 \mathrm{C}(5), 0$ GA $(30,6)$

CAM"ION LDUT

E?UIVALENCE ( LOIT(1),IU), (LOUT(2):hOLA(1).

I(LU.:T(11), OALT(1,1,1), (LOUT(3)1),FUIL(1,1,1)),

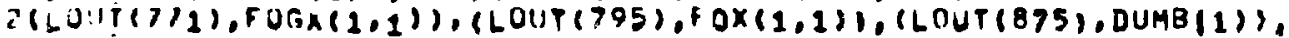

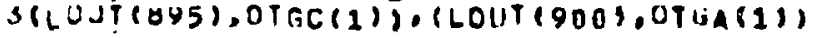

UTHENSIUN ERASE 350 .')

CAM'UU! ERASE

UIMENSIUN SSCI10),SI(10),SIFAHS(10),SIGINI10),SIGX(10):C(10), $1 A:(10), 50(10), P R O 3(.5,10), 51 G Y(16,54), 6 N U(19,54), 5) G A(18,54)$.

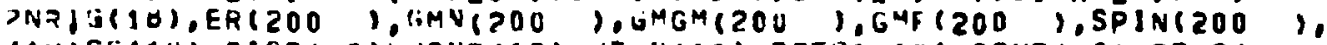
SAUASS(1甘), SIGP(1A): JONE (18), JTWU(18), DZERS(18), DONE(18),0T:0(18), It TA $(2 U:, 3(20), F 5: 34), F \times(20)$, DEL $(54), F(20,54), M$ DNE $(18), P(16,54)$, :PF(18,24),PZ(1001:,F21(1001),A1(18),EGK(55), TEMP(18), SIGEFY(IB).

C. $\operatorname{cr}(15,10)$

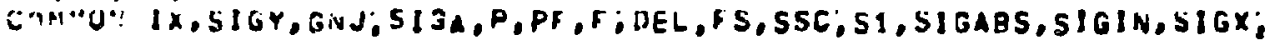
IC, A : SJ,PHOH, NAIU,Eh, JMH,GMFM,GMF,SPIN,AMASS, SIGP, JJNE, JTWJ.
$P T H_{L}$

PTHL

PTHL

PTBL

PTHL

PTBL

PTBL

PTHL

PTHL

PTHL

PTBL

PT는

PTHL

PTEL

PTHL

PTHL

PTHL

PTBL

PYRL

PTEL

PTRL

PTEL

PTHL

PTHL

PTEL

PTHL

PTHL

PTUL

PTEL

PTAL

PTEL

PITL

PTPL

PTHL

PTEL

PTHL

PTPL

PTHL

PTPL

PTEL

PTEL

PTFL

PTHL

PTrel

PTHL

PTHL

PTHL.

PTHL

PTEL

PTHL

PTEL

PTEL

PTHL

PTEL

PTHi 
2UTERU, UUNE, DTWO,ETA,O.ESC, MDOAE,KEY,MCURR, PZ,PZ1, RELSIG,A1,EBK:

PTHL STEMP, SIGEF Y, NDUNE

DIMENSIUN DATA $(165), 02(18), G R P(20), S O R(20), T(20,20), X T(20), V 1(20)$, I $V>(20), V 3(20), V A(20), V 5(20), C P(20), C N(20), C P E(20), C V 2(20), V(21)$, 2F $1(20), 02(20), 52(20), 31(20), 00(20), 10(20), 11(20)$

EJUIVAKENCE (EHASE(1),DATA(1),UZ(1),OLRP(1)).

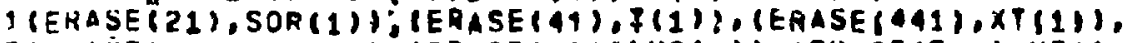

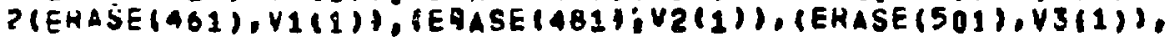

3(EKASE (521), VA(1)); (ERASE (541);V5(1)), (ERASE (562), EP(1)),

- (ERASE (581), CN(1)), (ERASE 1601$)$;CP2(1)), (ERASE 621$)$,CN2(1)),

S(EHASE (641), Y(1)), (ERASE (441), F 2(1)), (ERASE (461),F2(1)).

U(ERASE (AG1), S2(1)):(ERASE 501$), 01(1))$, (ERASE $(481), 00(1))$.

TERASE (401), TO(1), (ERASE (441),T1(1))

EOUIVALENCE (FX(1); ISD(1), (CF(1), CONCTA(1)), (LIB2,NL1日2)

COMADV NLIB

COMMOV/CADY/CDYCI1OI, IGCDY, ICALY

IGCUY $=16$

$S I G P L=S I G P(J)$

$J \in=J \operatorname{Jint}(J)$

If (J1) $<6,10,20$

1 ii $S\left\{G^{\prime \prime 1} 1=l\right.$.

bागSi

2. SIU"1=SIGP(J1)०DOVE(J) /DZERO(J)

उᄀ $7 x=7 t h u(j)$

IF(J2) $56,40,56$

4 : 5 I $422=1$.

GnTण

50 SIG:42=SIGP(JZ)

6: $S I G T=(S) G P Z+5 I G 4 I+S I G 42)$ DZERO(J)

$F L X=U$.

Vil $=1$,

Uก1 $10 . v=1, N x$

Q $\rightarrow 11)=0$.

$M=191 \square($ ( N )

$\operatorname{IF}(C T(J, M)) 80,70,90$

7ก $x T(V)=\xi S C(M)$

IF(IG.GE.53,AND.JUAOY.ED.1) XT(N)=CDYC(M)

कीTग11日

85 $X T(Y)=S I G T$

UTY JNRE1, NRX

IF ( \% VKUP(NR) )100.110.90

oO Cni:TIVUTE

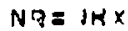

10 " H.AAXIHT(NR)

Uר $(\cdot)=\operatorname{VUL}(N)$

$V \eta=V U+V U L(N)$

$13 i$ F $F L X=F L X+V O L(N) \bullet F(V, I G)$

11 i coritiog

I5(:HIU(J)):60.160, CC!

16.) CALLP7LALE

$P !)=$.

$P_{1}=\ldots$.

Un1 $20: v=1.4 x$

(F) JU(i)) 170.160.17.

$1707=50+10(14)$

PTHL

PTHL

PTHL

PTBL

PTBL

PTBL

FTHL

PTㄴ

PTHL

PTHL

Рт甘L

PTEL

PT甘L

PTHL

HAMM

HAMM

PTEL

PTBL

PTBL

PTEL

PTEL

PTHL

PTHL

PTHL

PTRL

PTHL

PTBL

PTEL

PTBL

PTHL

PTHL

PTBL

PTBL

PTHL

PTHL

HAMM

PTEL

PTBL

PTHL

PTHL

PTEL

PTEL

PTEL

PTHL

PTHL

PT甘L

PTHL

PTEL

PYHL

PTHL

PTEL

PTHL

PTEL

PTHL 


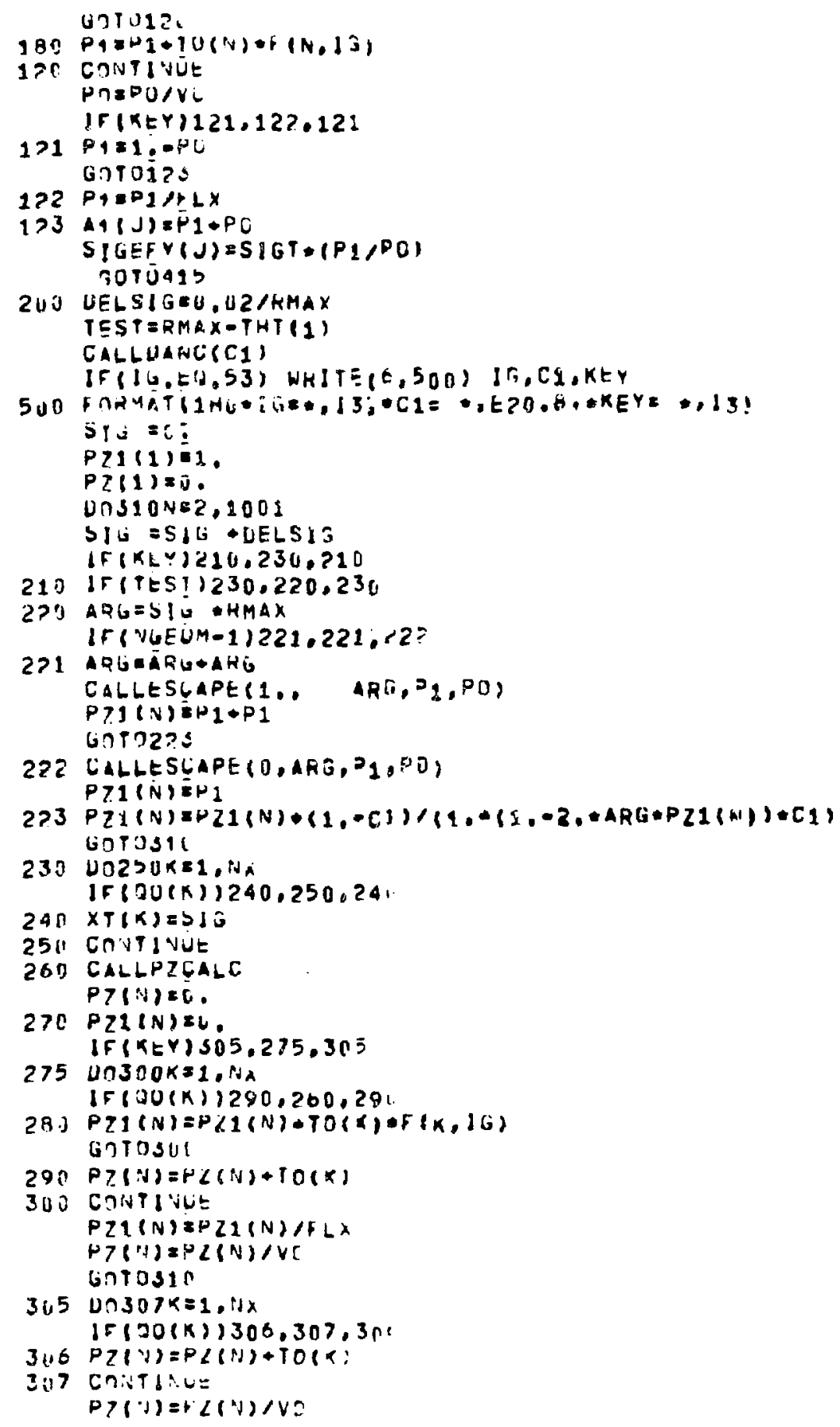

PTH

PTE

PTP

PTE

PTE

PTE

PTE

PTE

PTE

PTE

PTH

PTE

PTE

PTE

PTE

PTF

PT

PTE

PTE

PTE

PTE

DTE

PTH

PT

PTE

PTE

PTt

PTt

PTH

PT

PT

PT:

PIt

PTI 
SIIGHUJIINE INANCIC1)

C)AHC

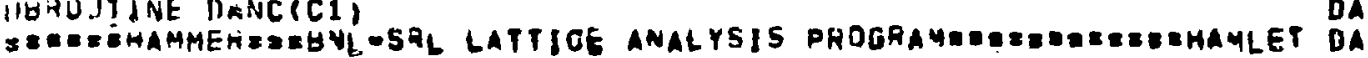

CDAHC DAFCUFE CORHECTIOV TOO SINGLE RODS

UIMENSIUN CNTRL $(2001,0 T$ TL19),LNK\{10)

COMMOV CNTAL

EOUIVALENCE

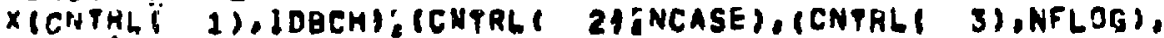

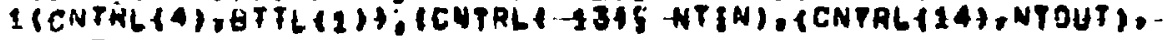

? (CNTHLI 15).NTPUN): (CYTRL 16) ONCHAIN). (CNTRLI 17).6IB1).

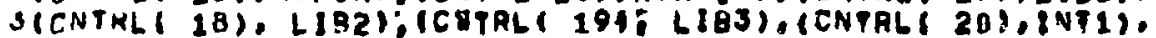

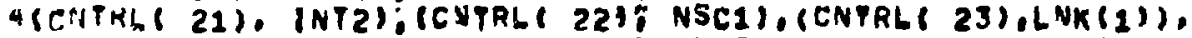

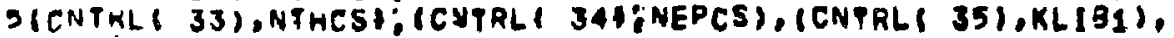

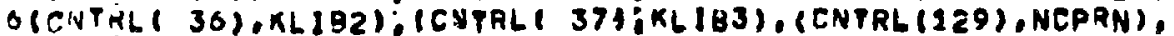

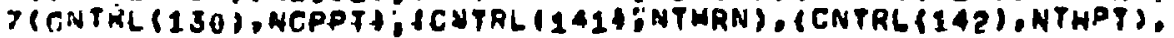

H(CNPHL (143), NTMPN): (CNPRL (153) (NEPRN), (CNTRL(154) .NEPPT).

O(CNTHL (155). NEPPN): (CNTRL (1564:NEPSG). (CNTKL(157).NEPAG).

$\triangle$ (C.JPHL (165). NFGRN): (CNTRL 1 166):NFGPT), (CNTRL(167), NFGPN).

J(CNTHL (168). NFGPU): (CYTRL(169); NFGJO) , (CNTRL (177) . NEDRN).

C (CINTRL (178), HEDPT): (CNTRL (170):NEUPN), (CNYRL (180).NEDNE),

"(CNTHL (181),NEDFW): (CHPRL(18C):NEURE), (CNTRL(183) ,NEDAX).

E(CNTRL (184), NE UVU)

UIMENSIUN LIAP(60O),NDLI9), NPTI20), THT(20), MXAS(20),

IILNI (20), NRHP (20) ODEGC (20), HSTHA (18), WSTBB (16),

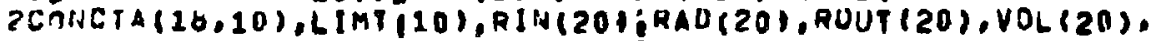

JMTUL(20), HEGV(20) Of ISD(PO),HOL U (3,1B)

LOM.1OV LIMP

ETUIVALENCE

J ILFH 1$).$

र(LI"s) 4).

J(LI"Mp( 7$)$.

4(Ld"pe 10$)$.

s(liat ?1).

IDEYTI, ILIMPI 2$\}.$ NPXI:PLIMPI 51.

NGESMI,ILIMP( 8$)$.

NKPI.PLIHPI 111.

NPTI1), ILIMPI 411.

lLm)(1)i, ILIMP(101)

NREP(1)) (LIMP(121):

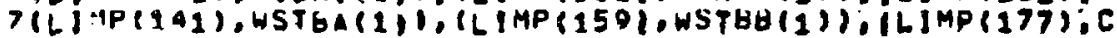

O(LIMP(S5)), LIMTII) ILIIMP(367).

U(LJHP(AO) . HOUT1211,1LFMP(427).

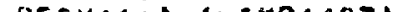
$R(N(1)),(6)$ MP $(337)$.

VOL(1)):ILIMP(447):

NX):(LIMP( 3$).$
(SOX):ILIMP( 6$):$ NP1B1), (LIMP( 9$).$ BSOD), (LIMP( 12$)$. THT(1)). (LIMP( 61 ): $(16,4,5): F 014(20,4,5):$

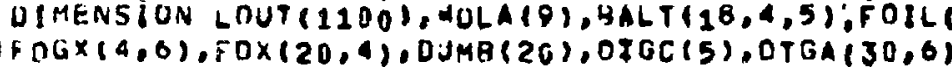

COMMUPS LOUT

EJUIVALENEE ( LOIT(1),ID), (LOUT(2).HOLA(1)),

: (LUJT(11),BALT(1,1;1), (LOUT $(371), F O I L(1,1,1))$,

? (LOHT(771),FOGX(1,1)), (LOUT (795),FOX(1,1)), (LOUT(875),DUMH)1),

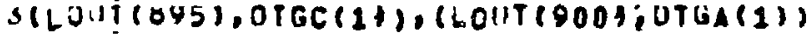

DI MENS IUN ERASE 350 (

CIM*UN $E R A S E$

D)MENSIUN SSII10),S1(10),SIRAAS(10),SIGIN(10),SIGX(10):C(10), $14 \cdot(10), 50(10), P Q 03(25,10), 5(G Y\{16,54), G N U(18,54), 5(G A(18,54)$,

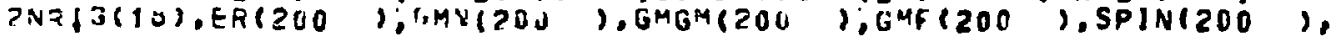

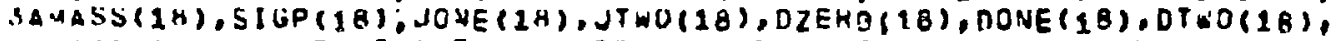
At $P$ A $(2 \mathrm{j}), 2(20), F S(54), F \times(20)$, DEL $(54), F(20,54), M D O N F(18), P(10,54)$,

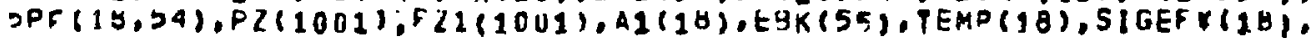

$\therefore \operatorname{CT}(10,10)$

CAN'UU JX,SISY,GNJ;SISA,P,PF,F;UEL,FS,SSC,SI, SITARS, SIGIN,SIGX;

IC, $\because$, S., OKUL, NKIG,EF, SMM, GMTM, GMF, SPEN, AMASS, SIGP, JONE, JTHJ,
DA

DA

DA

DA

DA

DA

DA

DA

DA

DA

DA

DA

DA

DA

DA

DA

DA

DA

DA

DA

DA

DA

DA

DA

DA

DA

DA

DA

DA

DA

DA

DA

DA

DA

DA

DA

DA

DA

DA

ni

D.

DA

DA

DA

D.

01

DA

D)

D.

DI

DI

I.

DS 
¿OZE $\%$, UUNE, DTHO,ETA,O,ESC, MDONE, KEY, MCURH,PZ,PZ, DELSIG, A1,EQK

DANC STEHP, SILEFY, NDONE

$$
-\cdots
$$

UTMENSIUN DATA $(165), 02(18), G R P(20), S O A(20), T(20,20), X T(20), V 1(20)$,

IVS(20), V3(20), VA(20), V5 (20),CP(20),CN(20):CP2(20),CN2(20), $(21)$,

PF $1(20), F 2(2),, S 2(20), 01(20), 00(20), T 0(20) ; 71(20)$

EJUIVALENCE IEMASE(1).DATA(1).DZ(2),GRP(1)!:

TERASE (21), SOA (1) i, (ERASE(41), F(1) ), (EKASE (441),XT(1)):

2(ERASE (4O1),V1 (1), (ERASE (481),V2(1)), (ERASE(501), V3(1)),

S(ERASE $(S 21), V A(1)),(E Q A S E(541)$,VS(1)), RERASE $(561), C P(1))$,

- (ERASE(SB1),CN(1)), (EQASE $(601)$ :CP2 (1)), (ERASE $(621)$,CN2(1)),

S(FRASE (O41), Y (1)), IERASE (449), (1)(1)), (ERASE $(461), F 2(1)$ ).

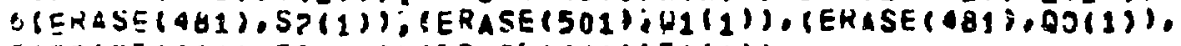

TERASE (461). TR(1)): (ERASE(441):T2(1))

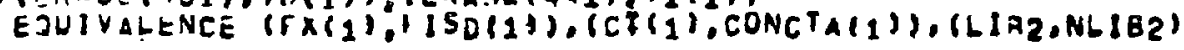

COMHUN: NLIE

COPION/CAOY/CDYCI10I, IGCDY, ICAUY

irsigitis

|f $\mid \cdot N x+1 / 200,200,1$

$2 N X=\cdot 3 x$

IF :IGEUM-2)16.10,2c

1) $C>C .9323126:$

C.3ะ11.12

GกTกj

$>C>0.9 .802260^{\circ}$

C3×ก:1:

35 Lna 2 No! $(1)+1$

$S_{L}=\dot{S} .1$

If (LUN+WX) 35.35.45

35 Uาด IIN=LUN, NX

$M=$ P.

IFISL,LI,53.0R, ICAOR, VE, 1)

I $S_{L}=S L$ - (HOUT(V)-RJUT(NA1)H+(SSC(M)+SIGIV(M)+SIGABS(M))

IF (IU. GE.53.AND. ISADY.ED,1)

ISL ESL + IKOUT (N)-ROJTIN-ITIN (CDYC(M).

'SIGIV(M) SIGARS(M))

43 COIT, Vut

45 If $($ JUEUM-1)7r, 70.73

7.) $S_{L}=S_{L}+2$.

1F(SL-1.)71,71.72

$71 C 1=1,-S L=(2,+S L+(A L O G(5 L)-.9228-S L+(.332-.0354+5 L))$ Gก1J111

. $C$ CIEXD) $-S L)+(2 .+0.16 /(S L+3)+2) /.(S L+3$. inTa111

is condtivie

$V$ HUF $=\left(\right.$ KOUT $(N X) / R O \perp T\left(L B_{n-1}\right)$

$S L=S L=2 .-(Y M O F+1$,

$V \cdot 4 O F=V M U F+2-1$.

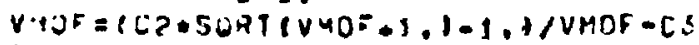

If (VM) IS1.51.5?

52 CDSUM:. $5 i$

$15122-20.150 .60 .00$

$51 \mathrm{C}>=.$.

5: $C,=E x=(-25) /(1,+5+-5 z)$

3)

69 $\ddot{*}=$.

DANC

DANC

DANC

DANC

DANC

DANC

DANC

DANCC

DANC

DANC

DANC

DANE

DANC

DANC

HAMHEH

HAMMER

DANC

Danc

DANC

DANC

DANC

DANC

DANC

DANC

DANC

DANC

DANSC

DANC

DANC

HAMMER

HAMMER

HAMMER

HAMMER

HAMMER

HAMMEF

Daric

Danc

Dave

EAPUC

DANEC

DANC

DANC

DANC

DANC

DANC

DA INC

DANC

DA'TC

Dane

DANC

DA.JC

DA V C

DA:IC

DA: C 
GROUTINF DARE

CUC 6000 FTN V3:002261 UPTE2 11/21

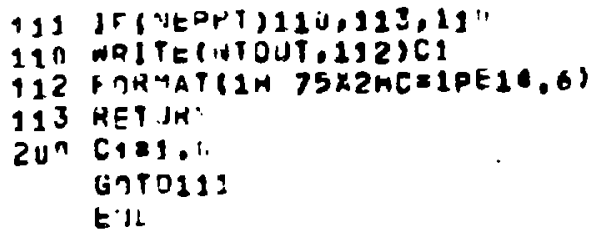


SRIE

SIHAUU!l VE GFIU (:U,)

Qin 10

riviRJTES MESH VE OOEU FOK CROSS SECTIOH CALCULATIOH,

GNIS

QN:D

DIMENSIUN DUMY(1900)

6in 10

COM.OUN UUMY

ANID

E TUIVALENCE (NOUT, NUFY 121 1"

GA10

an 10

UIMENSIUN

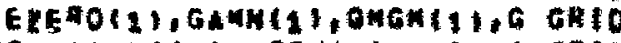

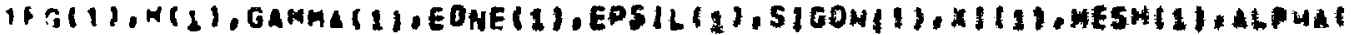

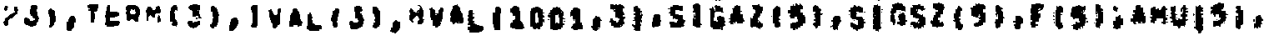

\$10

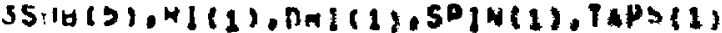

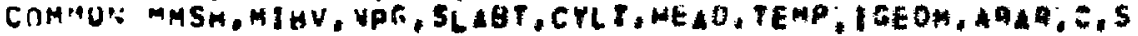

Cat 10

010

GAID

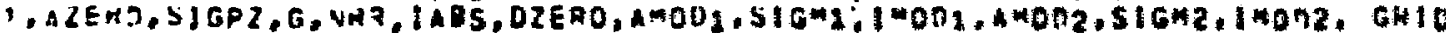

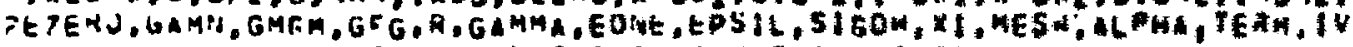

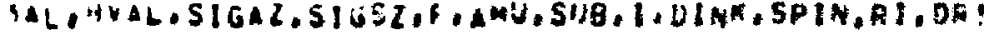

$\therefore, T A D S, H H A R, 52,53,54,11,72, T 3,14$

CAM"UVJJJD/ERIL.

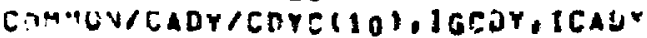

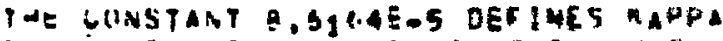

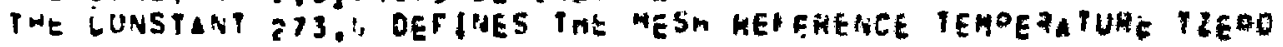

$n+x=:$

$S \perp P=>$

M1 U. E104E-5.273,0/12ERA

$\forall 31,1 / 1(310 A 2 E k n \cdot 4 Z R 3)$

$J \rightarrow=:$

$I S=\mathrm{don}$

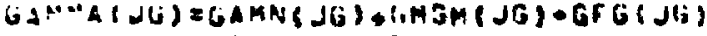

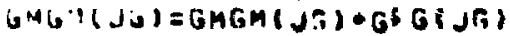

GFU(Ji) = SFG(JG)/G*G!'JG)

IF( $7(\mathrm{Ju})) T 0,70.6 \mathrm{r}^{\circ}$

6: $\operatorname{kP}=4(J)$

(i) $T \mathrm{~T}>\mathrm{S}$

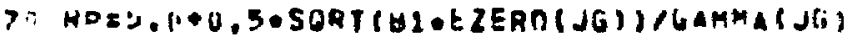

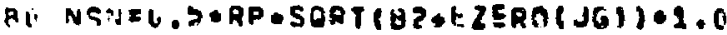

E.:S:I: VI:

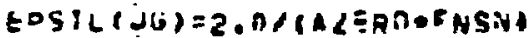

IF (IGEUY,EU.53) EOSILPJO)=0.5०ERSIL(JE)

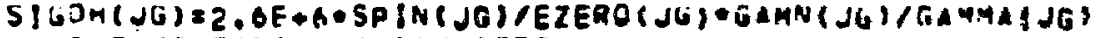

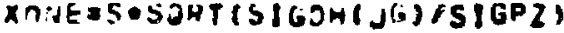

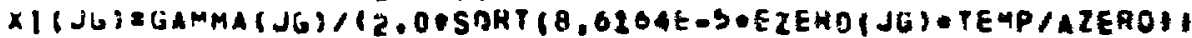

If $(12: 0 / x \mid(\mid f)=x) \operatorname{lot} / 2.0) 130.103,220$

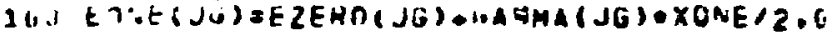

$\operatorname{SAR}=771.1$

(G) IU 140

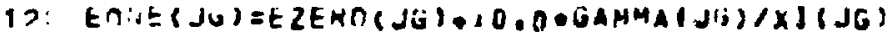

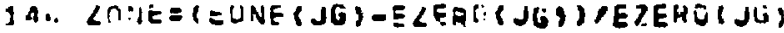

CIITEAMAXIIEPILLIEZENOPJB).0.42637142057)

IS (COT.E- (1.,1)-CUT) 160.100,10.2

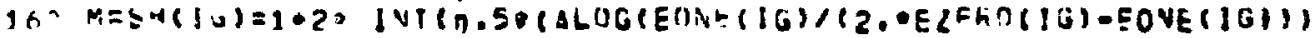

1 (EPSIL(IG) ),

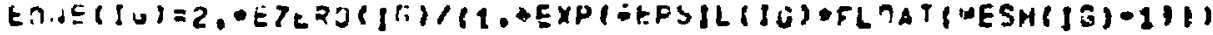

G) 10 ¿थं

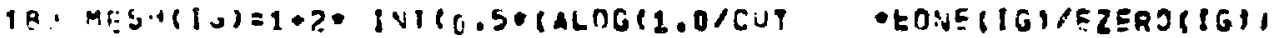

610

GAID

GA10

onI

MANHER

GH10

GA 10

Ga 10

onto

GHID

Q⿻川 10

Q⿻日土龰D

GA10

GHIH

6h1 10

Gats

GA 10

AF 10

GH1D

Gato

GAID

Q⿻土一𧘇 10

on 15

GNID

CE 10

MAMHE

SH 10

GR 10

GR 10

GH:D

GR 10

SRIV

GAID

GRIO

GN1D

$\sin 10$

GR 10

GK!D

GeIU

Gin ID

Sक्राD

G) 10 


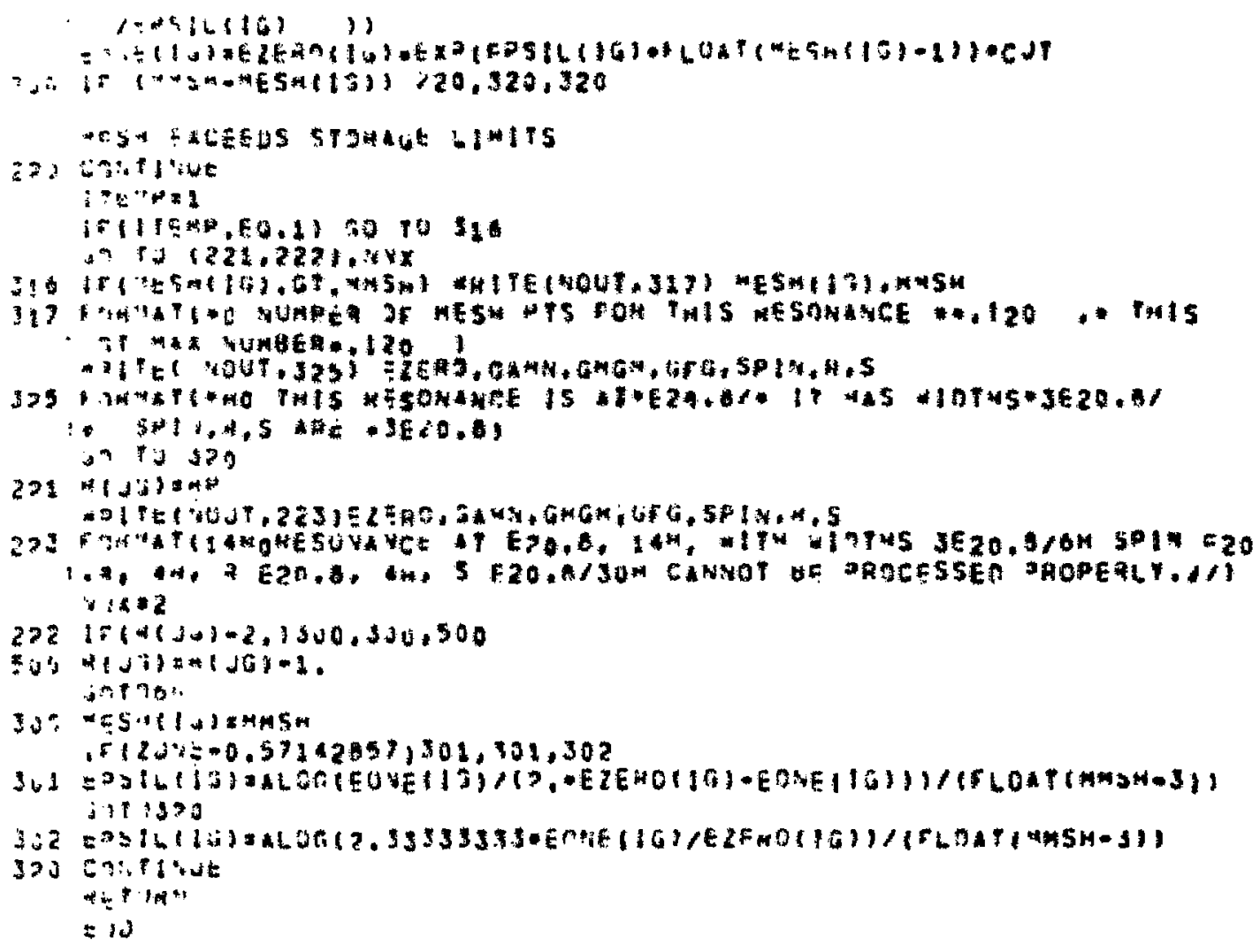




\section{REFERENCES}

1. J. E. Sulch and H. C. Honeck, "The HAMER System - Heterogeneous Analysis by Mulcigroup Methods of Exponen ials and Reactors", DP-1064, Savannah River Laboratory (1964).

2. S. Jabtawy, J. Karnl, W. Rothenstein and S. Velner, "Water-Moderated Rcaccor Analysis wth ENDF/B Data", In Nuclear Data in Science and Technoloky, Vol. II, proceadings of a symposium, Paris, March 12 to 16, 1973, International Atomic Energy Agency (1973), paper IAEA-SM-170/8.

3. K. B. Cady, G. J. Kirouac and J. J. McInerney, Nuc1. Sc1. and Eng. 29 (1967).

+. N. Corngold, Ann. Phys. 11, 338 (1960).

5. M. M. R. Wllliams, The Slowing-Down and Thermalization of Neutrons, John Hiley and Sons, Inc., New York (1966), pp. 97 ff.

6. J. Mardy, Jr., Nurl. Sc1. and Eng. 27, 135 (1967).

7. Archur Buslik, "Calculational Model for the Resonance Absorption in the Pu 246 i eV Resonance", BNL-19046/RP-1034, Jun' 1974.

b. Arthur Buslik, "Proton Chemical Binding in Water and ies Effects on Neutron Absorption in the 1 eV Resonance of Pu-240', BNL-19411.

9. Sec pare 58 of reference $>$.

10. M. Nelkin, Phys. Rev. 119,741 (1960).

11. D. Parks, Nucl. Scl. Eng. 9, 430 (1961).

12. L. Finkelstein and M. Shatz, Nucl. Sci. and Eng. 42, 260 (1970). 


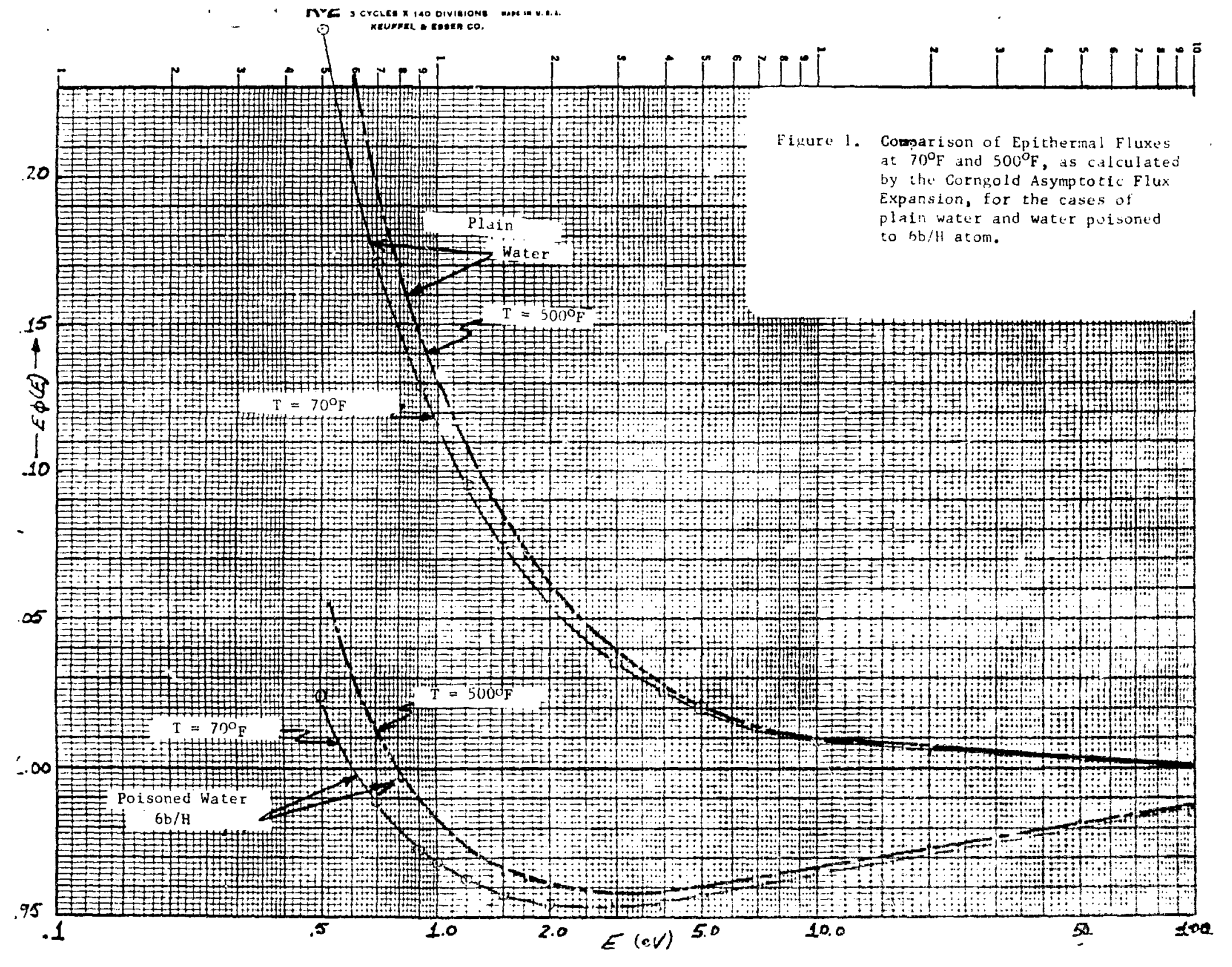




\section{neurre- cosen co.}

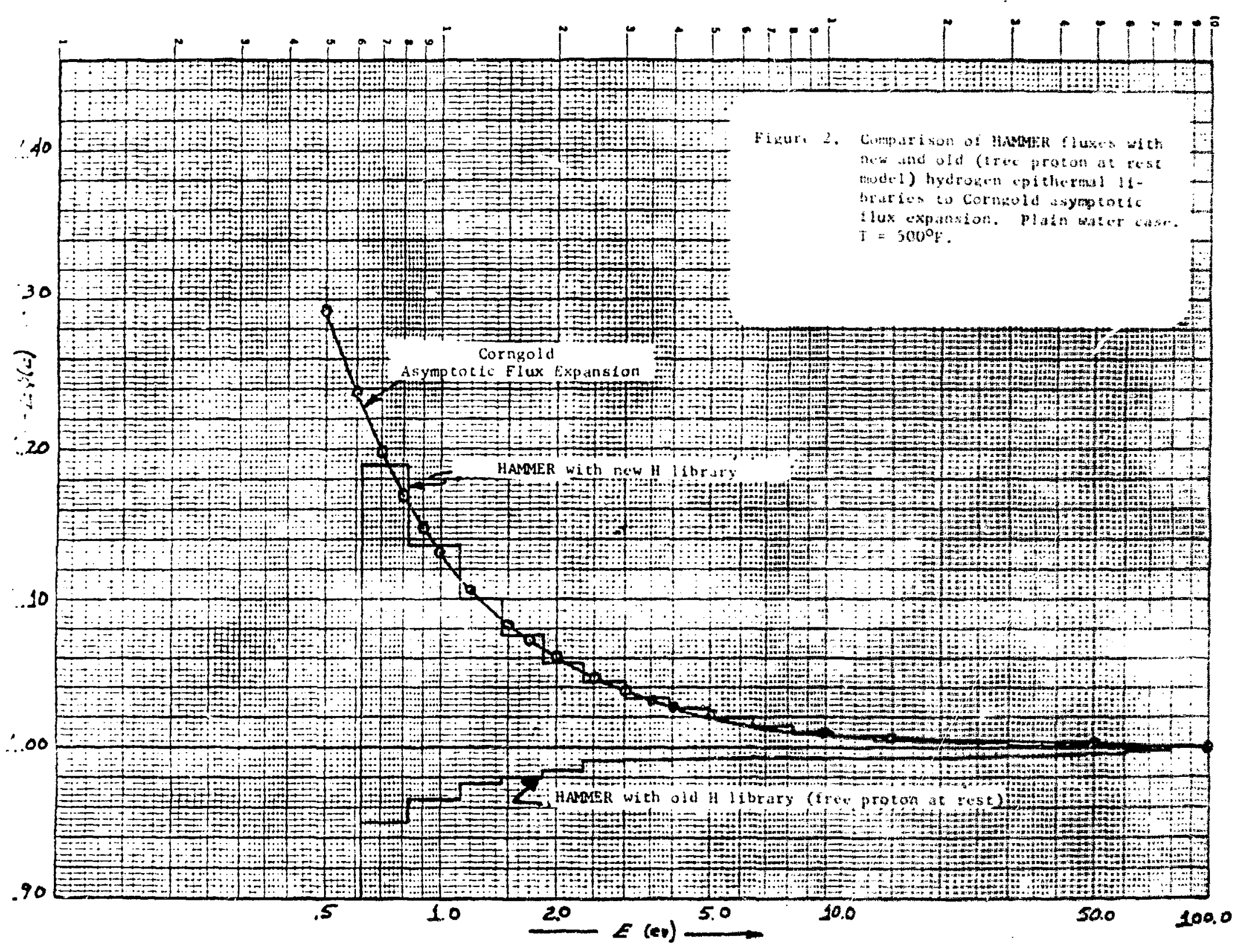




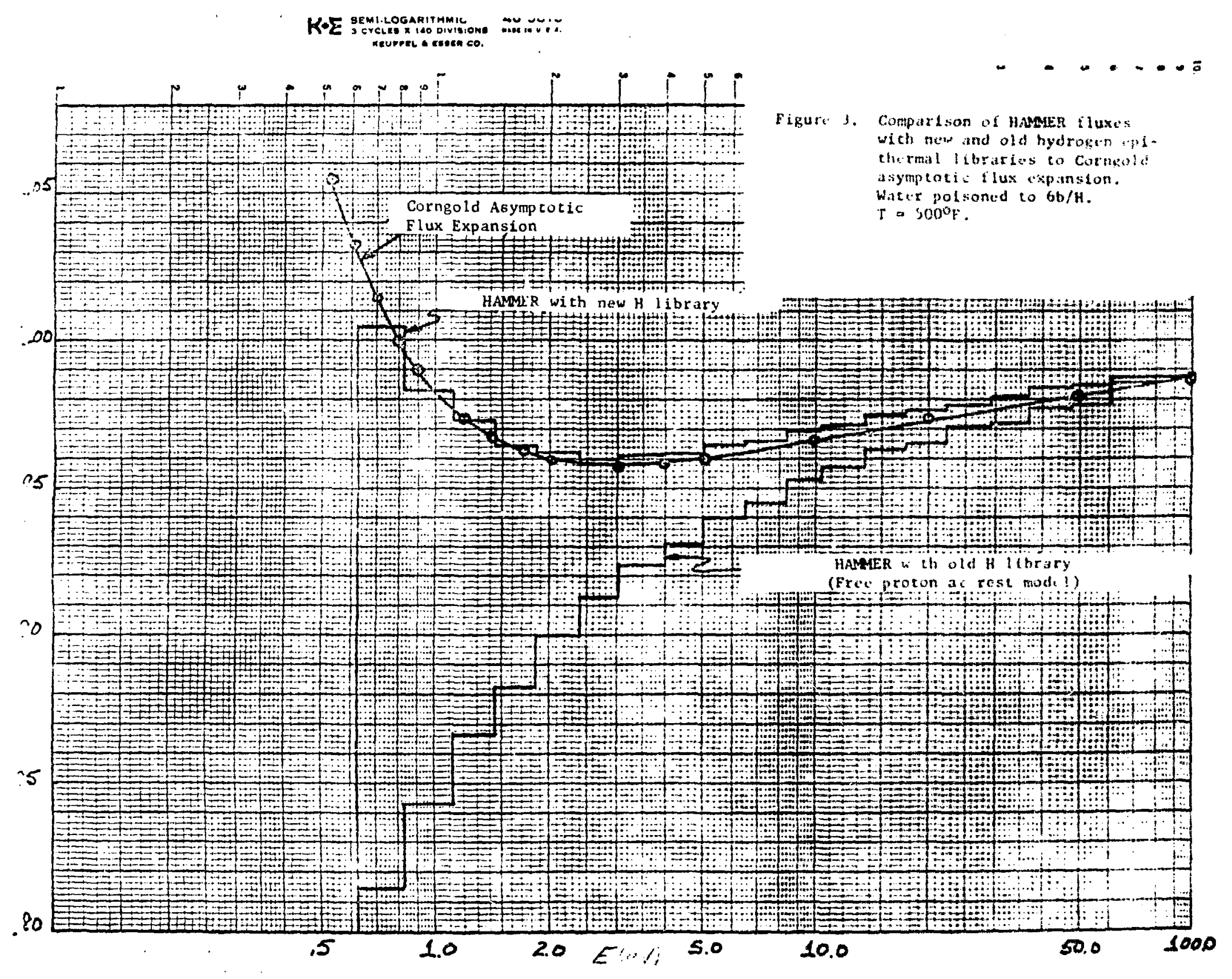

